\title{
THE RANDOM-CLUSTER MODEL ON THE COMPLETE GRAPH
}

\author{
Béla Bollobás, Geoffrey Grimmett, Svante Janson
}

\begin{abstract}
The random-cluster model of Fortuin and Kasteleyn contains as special cases the percolation, Ising, and Potts models of statistical physics. When the underlying graph is the complete graph on $n$ vertices, then the associated processes are called 'mean-field'. In this study of the mean-field random-cluster with parameters $p=\lambda / n$ and $q$, we show that its properties for any value of $q \in(0, \infty)$ may be derived from those of an Erdös-Rényi random graph. In this way we calculate the critical point $\lambda_{\mathrm{c}}(q)$ of the model, and show that the associated phase transition is continuous if and only if $q \leq 2$. Exact formulae are given for $\lambda_{\mathrm{c}}(q)$, the density of the largest component, the density of edges of the model, and the 'free energy'. This work generalizes earlier results valid for the Potts model, where $q$ is an integer satisfying $q \geq 2$. Equivalent results are obtained for a 'fixed edge-number' random-cluster model. As a consequence of the results of this paper, one obtains large-deviation theorems for the number of components in the classical random-graph models (where $q=1$ ).
\end{abstract}

\section{Introduction and summary}

The Potts model is one of the standard processes of statistical physics. When the underlying graph is complete, the ensuing process is termed 'mean-field'; unlike the corresponding lattice models, the mean-field Potts model is exactly soluble (see Wu (1982), Kesten and Schonmann (1990)). Intimately related to Potts models are random-cluster models, whose discovery was reported in a series of papers by Fortuin and Kasteleyn around 1970 (see Grimmett (1994a, b) for a survey and historical account). The subject of the current paper is a 'complete' analysis of random-cluster models on complete graphs, i.e., the mean-field random-cluster model.

The model in question is as follows. We shall consider graphs on a set $V$ of $n$ vertices, and so the edge-sets of our graphs will be subsets of $V^{(2)}$, the set of $\left(\begin{array}{l}n \\ 2\end{array}\right)$ pairs of elements of $V$. Let $0<p<1, q>0$, and for $E \subseteq V^{(2)}$ define

$$
\widetilde{P}(E ; n, p, q)=p^{|E|}(1-p)^{\left(\begin{array}{c}
n \\
2
\end{array}\right)-|E|} q^{c(V, E)}=(1-p)^{\left(\begin{array}{c}
n \\
2
\end{array}\right)}\left(\frac{p}{1-p}\right)^{|E|} q^{c(V, E)},
$$

1991 Mathematics Subject Classification. 05C80, 60K35, 82B20.

Key words and phrases. Random-cluster model, mean-field model, phase transition, random graph.

This version was prepared on 6 September 1995. 
where $c(V, E)$ denotes the number of components of the graph $(V, E)$. Furthermore, let

$$
Z(n, p, q)=\sum_{E} \widetilde{P}(E ; n, p, q)
$$

and define the probability

$$
\mathrm{P}(E ; n, p, q)=\frac{\widetilde{P}(E ; n, p, q)}{Z(n, p, q)} .
$$

Thus, for every $n, p, q, \mathrm{P}(E ; n, p, q)$ is a probability measure on the set of possible edge-sets $E$; hence it describes the distribution of a random graph with $n$ vertices, which will be denoted by $G(n, p, q)$. We sometimes write $\mathrm{P}(E ; n, p, q)$ as $\mathrm{P}(E ; V, p, q) ;$ as is customary, $V$ is usually taken to be $\{1,2, \ldots, n\}$.

The random-cluster measure given in (1.3) has two parameters, $p$ and $q$. When $q=1$, we recover the usual Erdös-Rényi model $G(n, p)$; see Bollobás (1985) for the general theory of such a random graph. When $q \in\{2,3, \ldots\}$, the random-cluster model corresponds to a Potts model on the complete graph $K_{n}$ with $q$ states and with strength of interaction $J=-\log (1-p)$. Indeed the phase structures of the two models are closely linked; see Edwards and Sokal (1988) and Grimmett (1994b). Whereas Potts models are defined for integer values only of $q$, the random-cluster model is well defined for all real positive $q$. In this sense, random-cluster models generalize Potts models. Professor Kasteleyn has pointed out that they are more general than Potts models in a further regard: there are questions which may be asked about random-cluster models for which there is no corresponding question for Potts models (see Grimmett (1994a)). Material related to the current paper may be found in Whittle $(1986,1994)$.

The principal technique for analysing the mean-field Potts model relies upon the assumption that $q$ is an integer. This technique is invalid in its basic form for general real values of $q$, and therefore one needs extra methods in order to understand random-cluster models. Our principal extra technique, described in Section 3 , is a method whereby properties of $G(n, p, q)$ may be studied via corresponding properties of the usual random graph $G(n, p)$. It is striking that our results are valid for all $q>0$, including the regime $0<q<1$; when $0<q<1$, the FKG inequality is no longer valid, and consequently there is a problem in studying the phase transition in lattice systems (see Grimmett (1994b)).

Our principal results concern the process $G(n, p, q)$ defined above. There are complementary results for a 'fixed edge-number' model, and we sketch these later in this section; see also Section 11.

As in the Erdös-Rényi theory of the giant component in the case $q=1$, we set $p=\lambda / n$ where $\lambda$ is a positive constant, and shall study the size of the largest component of the ensuing $G(n, \lambda / n, q)$ in the limit as $n \rightarrow \infty$. It turns out that there is a critical value of $\lambda$, depending on the value of $q$, which marks the arrival of a 'giant component' of the graph. This critical value is given by

$$
\lambda_{\mathrm{c}}(q)= \begin{cases}q & \text { if } 0<q \leq 2 \\ 2\left(\frac{q-1}{q-2}\right) \log (q-1) & \text { if } q>2\end{cases}
$$


Another quantity having a central role is the function $\theta(\lambda, q)$ given as follows. We define

$$
\theta(\lambda, q)= \begin{cases}0 & \text { if } \lambda<\lambda_{\mathrm{c}}(q) \\ \theta_{\max } & \text { if } \lambda \geq \lambda_{\mathrm{c}}(q)\end{cases}
$$

where $\theta_{\max }$ is the largest root of the equation

$$
e^{-\lambda \theta}=\frac{1-\theta}{1+(q-1) \theta}
$$

It may be seen that $\theta(\lambda, q)>0$ if and only if either (a) $\lambda>\lambda_{\mathrm{c}}(q)$, or (b) $\lambda=\lambda_{\mathrm{c}}(q)$ and $q>2$ (a proof of this is given after equation (6.22)). Furthermore $\theta(\lambda, q)$ is nondecreasing in $\lambda$, and it follows that $\theta(\cdot, q)$ is continuous if $0<q \leq 2$, and has a unique (jump) discontinuity at $\lambda=\lambda_{\mathrm{c}}(q)$ if $q>2$. This jump discontinuity corresponds to the 'first-order phase transition' of statistical physics.

We shall prove the following (and more) in Sections 2, 4, and 9.

(a) If $0<\lambda<\lambda_{\mathrm{c}}(q)$ and $q>0$, then a.e. $G(n, p, q)$ has largest component having order $\log n$.

(b) If $\lambda>\lambda_{\mathrm{c}}(q)$ and $q>0$, then a.e. $G(n, p, q)$ consists of a 'giant component' of order $\theta(\lambda, q) n$, together with other components of order $\log n$ or smaller.

(c) If $\lambda=\lambda_{\mathrm{c}}(q)$ and $0<q \leq 2$, then a.e. $G(n, p, q)$ has largest component of order $n^{2 / 3}$.

(d) If $\lambda=\lambda_{\mathrm{c}}(q)$ and $q>2$, then a.e. $G(n, p, q)$ is either as in (a) or as in (b).

In the above, we say that 'a.e. $G(n, p, q)$ satisfies property $\Pi$ ', for a given sequence $p=p(n)$ and a fixed $q$, if $\mathrm{P}(G(n, p, q)$ has $\Pi) \rightarrow 1$ as $n \rightarrow \infty$. Care must be taken in interpreting the above remarks concerning the orders of components. See Theorems 2.1-2.3 for more precise statements.

There are two main steps in establishing the above facts. The first step is to establish the relation (1.6) by studying the size of the largest component of $G(n, \lambda / n, q)$. When $q>2$, the relation (1.6) has three solutions for large $\lambda$; in order to decide which of these is the density of the largest component, we shall study the number of edges in $G(n, \lambda / n, q)$. That is to say, we shall find the function $\psi(\lambda, q)$ such that a.e. $G(n, \lambda / n, q)$ has (order) $\psi(\lambda, q) n$ edges. It is interesting to note that, at the critical point of a first-order phase transition, the function $\psi(\cdot, q)$ is discontinuous.

We present in Theorem 2.6 the asymptotic behaviour of $n^{-1} \log Z(n, \lambda / n, q)$ as $n \rightarrow \infty$; this amounts, in physical terms, to a calculation of the 'free energy'. As a consequence of this calculation, we obtain large-deviation results for the number of components of $G(n, \lambda / n, q)$ for $q>0$ (see Section 8).

Another random-graph model of interest is that with a fixed number of edges; the corresponding random-cluster model $G(n, m, q)$ is given by attaching weight $q^{c(E)}$ to each edge-set $E$ of $K_{n}$ having cardinality $m$, where $m, n$, and $q$ are fixed in advance. This model is also obtained if we condition $G(n, p, q)$ (for any $p$ ) on having exactly $m$ edges. For $G(n, m, q)$ one may obtain results similar to those described above for $G(n, \lambda / n, q)$, so long as $m$ and $n$ are related asymptotically in the manner of $m / n \rightarrow \mu$ for some positive constant $\mu$. Some of these results depend on a certain hypothesis of which we have no complete proof (see Hypothesis 11.5). Note that 
the two models cannot behave (asymptotically) identically, for the following reason at least. The asymptotic number of edges per vertex in a.e. $G(n,\lfloor\mu n\rfloor, q)$ is exactly $\mu$, where $\mu$ may take any value in $(0, \infty)$; on the other hand the corresponding 'edge-density' $\psi(\lambda, q)$ of $G(n, \lambda / n, q)$ has a jump discontinuity at $\lambda=\lambda_{\mathrm{c}}(q)$ when $q>2$, and therefore may not take all values in $(0, \infty)$.

The paper is laid out as follows. In Section 2 we state our main results for $G(n, p, q)$ under the assumption $q>0$. This is followed in Section 3 by an account of the central technique of the paper. Proofs of main results when $q \geq 1$ appear in Sections 4-6. In Section 7, we summarise facts about the nature of the phase transition, and in Section 8 we describe an application of one of our calculations to the large-deviation behaviour of the number of components of a random graph. Similar conclusions are valid when $0<q<1$, as stated in Section 2; see Section 9. An external field is added in Section 10. Finally, Section 11 contains a brief adaptation of the arguments and conclusions to a 'fixed edge-number' randomcluster model $G(n, m, q)$.

\section{Phase transition}

We state our main results in this section. Until further notice we assume that $q>0$, and we write $p=\lambda / n$ where $\lambda$ is a positive constant. We shall sometimes suppress explicit reference to $q$. We define $\lambda_{\mathrm{c}}(q)$ by

$$
\lambda_{\mathrm{c}}(q)= \begin{cases}q & \text { if } 0<q \leq 2 \\ 2\left(\frac{q-1}{q-2}\right) \log (q-1) & \text { if } q>2,\end{cases}
$$

and $\theta(\lambda)=\theta(\lambda, q)$ by

$$
\theta(\lambda)= \begin{cases}0 & \text { if } \lambda<\lambda_{\mathrm{c}}(q) \\ \theta_{\max } & \text { if } \lambda \geq \lambda_{\mathrm{c}}(q)\end{cases}
$$

where $\theta_{\max }$ is the largest root of the equation

$$
e^{-\lambda \theta}=\frac{1-\theta}{1+(q-1) \theta} .
$$

We shall need to study the properties of roots of (2.3) in some detail, but defer this until Lemma 2.5. For the moment we note only that $\theta(\lambda)=0$ if and only if (a) $\lambda<\lambda_{\mathrm{c}}(q)$, or (b) $\lambda=\lambda_{\mathrm{c}}(q)$ and $q \leq 2$.

We now state three theorems, dealing respectively with the subcritical case $\lambda<$ $\lambda_{\mathrm{c}}(q)$, the supercritical case $\lambda>\lambda_{\mathrm{c}}(q)$, and the critical case $\lambda=\lambda_{\mathrm{c}}(q)$. In the matter of notation, for a sequence $\left(X_{n}\right)$ of random variables, we write $X_{n}=\mathrm{O}_{\mathrm{p}}(f(n))$ if $X_{n} / f(n)$ is bounded in probability, which is to say that

$$
\mathrm{P}\left(\left|X_{n}\right| \leq f(n) \omega(n)\right) \rightarrow 1 \quad \text { as } n \rightarrow \infty
$$

for any sequence $\omega(n)$ satisfying $\omega(n) \rightarrow \infty$ as $n \rightarrow \infty$. Similarly, we write $X_{n}=$ $\mathrm{o}_{\mathrm{p}}(f(n))$ if $X_{n} / f(n) \rightarrow 0$ in probability as $n \rightarrow \infty$, which is to say that

$$
\mathrm{P}\left(\left|X_{n}\right| \leq f(n) / \omega(n)\right) \rightarrow 1 \quad \text { as } n \rightarrow \infty
$$

for some sequence $\omega(n)$ satisfying $\omega(n) \rightarrow \infty$. Convergence in probability is denoted by ' $\stackrel{\mathrm{P}}{\longrightarrow}$ '. 
Theorem 2.1. Subcritical case. Assume that $q>0$ and $\lambda<\lambda_{\mathrm{c}}(q)$. Then a.e. $G(n, \lambda / n, q)$ comprises trees and unicyclic components only. There are $\mathrm{O}_{\mathrm{p}}(1)$ unicyclic components with a total number of $\mathrm{O}_{\mathrm{p}}(1)$ vertices. The largest component of a.e. $G(n, \lambda / n, q)$ is a tree having order $\alpha \log n+\mathrm{O}_{\mathrm{p}}(\log \log n)$ where $\alpha^{-1}=$ $-\log (\lambda / q)+(\lambda / q)-1>0$. The number of edges in $G(n, p, q)$ is $\lambda n /(2 q)+\mathrm{o}_{\mathrm{p}}(n)$.

Theorem 2.2. Supercritical case. Assume that $q>0$ and $\lambda>\lambda_{\mathrm{c}}(q)$. Then a.e. $G(n, \lambda / n, q)$ consists of a giant component, trees, and unicyclic components. The giant component has $\theta(\lambda) n+\mathrm{o}_{\mathrm{p}}(n)$ vertices and $\lambda \theta(\lambda)\left\{q^{-1}+\left(\frac{1}{2}-q^{-1}\right) \theta(\lambda)\right\} n+\mathrm{o}_{\mathrm{p}}(n)$ edges. The largest tree has order $\alpha \log n+\mathrm{o}_{\mathrm{p}}(\log n)$ where $\alpha^{-1}=-\log \beta+\beta-1>0$ and $\beta=\lambda(1-\theta(\lambda)) / q$. There are $\mathrm{O}_{\mathrm{p}}(1)$ unicyclic components with a total number of $\mathrm{O}_{\mathrm{p}}(1)$ vertices. The number of edges in $G(n, \lambda / n, q)$ is $\lambda\left\{1+(q-1) \theta(\lambda)^{2}\right\} n /(2 q)+$ $\mathrm{o}_{\mathrm{p}}(n)$.

Theorem 2.3. Critical case. Assume that $q \geq 1$ and $\lambda=\lambda_{\mathrm{c}}(q)$.

(a) If $1 \leq q \leq 2$, then a.e. $G(n, \lambda / n, q)$ consists of trees, unicyclic components, and $\mathrm{O}_{\mathrm{p}}(1)$ components with more than one cycle. The largest component has order $\mathrm{O}_{\mathrm{p}}(n)$. The total number of vertices in unicyclic components is $\mathrm{O}_{\mathrm{p}}\left(n^{2 / 3}\right)$. The largest tree has order $\mathrm{O}_{\mathrm{p}}\left(n^{2 / 3}\right)$.

(b) If $q>2$, then a.e. $G(n, \lambda / n, q)$ is as in either Theorem 2.1 or Theorem 2.2.

We conjecture that Theorem 2.3(a) extends also to $0<q<1$, but we have not been able to prove this; see Section 9 .

More detailed asymptotics are available for $G(n, \lambda / n, q)$ by looking deeper into the proofs. We remark that, in the case of Theorem 2.3(b) when $\lambda=\lambda_{\mathrm{c}}(q)$ and $q>2$, we have not checked whether both possibilities given above actually occur (with positive probabilities in the limit). If we allow $\lambda=\lambda_{n}$ to depend on $n$, where $\lambda_{n} \rightarrow \lambda_{\mathrm{c}}(q)$ as $n \rightarrow \infty$, then one may see that, for $q>2$ and $0 \leq \pi \leq 1$, there exist sequences $\left(\lambda_{n}\right)$ such that a.e. $G\left(n, \lambda_{n} / n, q\right)$ is as in Theorem 2.1 with probability $\pi$ and is as in Theorem 2.2 otherwise.

The giant component, when it exists, has order approximately $\theta(\lambda) n$, where $\theta(\lambda)$ is given in (2.2) and (2.3). We now study the roots of (2.3); note that $\theta=0$ satisfies (2.3) for all $\lambda$ and $q$, and that all positive roots satisfy $0<\theta<1$.

Let

$$
f(\theta)=\frac{1}{\theta}\{\log (1+(q-1) \theta)-\log (1-\theta)\}, \quad 0<\theta<1,
$$

and note that $\theta(\in(0,1))$ satisfies $(2.3)$ if and only if $f(\theta)=\lambda$. We start with two elementary lemmas concerning the function $f$.

Lemma 2.4. The function $f$ is strictly convex on $(0,1)$ and satisfies $f(0+)=q$ and $f(1-)=\infty$.

(a) If $0<q \leq 2$, then $f$ is strictly increasing.

(b) If $q>2$, then there exists $\theta_{\min } \in(0,1)$ such that $f$ is strictly decreasing on $\left(0, \theta_{\min }\right)$ and strictly increasing on $\left(\theta_{\min }, 1\right)$.

Proof. If $t>-1$ then $(1+t \theta)^{-1}$ is a strictly convex function of $\theta$ for $0<\theta<1$; hence $f(\theta)=\int_{-1}^{q-1}(1+t \theta)^{-1} d t$ is also strictly convex. Furthermore,

$$
\lim _{\theta \uparrow 1} \log \left\{\frac{1+(q-1) \theta}{1-\theta}\right\}=\lim _{\epsilon \downarrow 0} \log \left\{\frac{q-(q-1) \epsilon}{\epsilon}\right\}=\infty,
$$




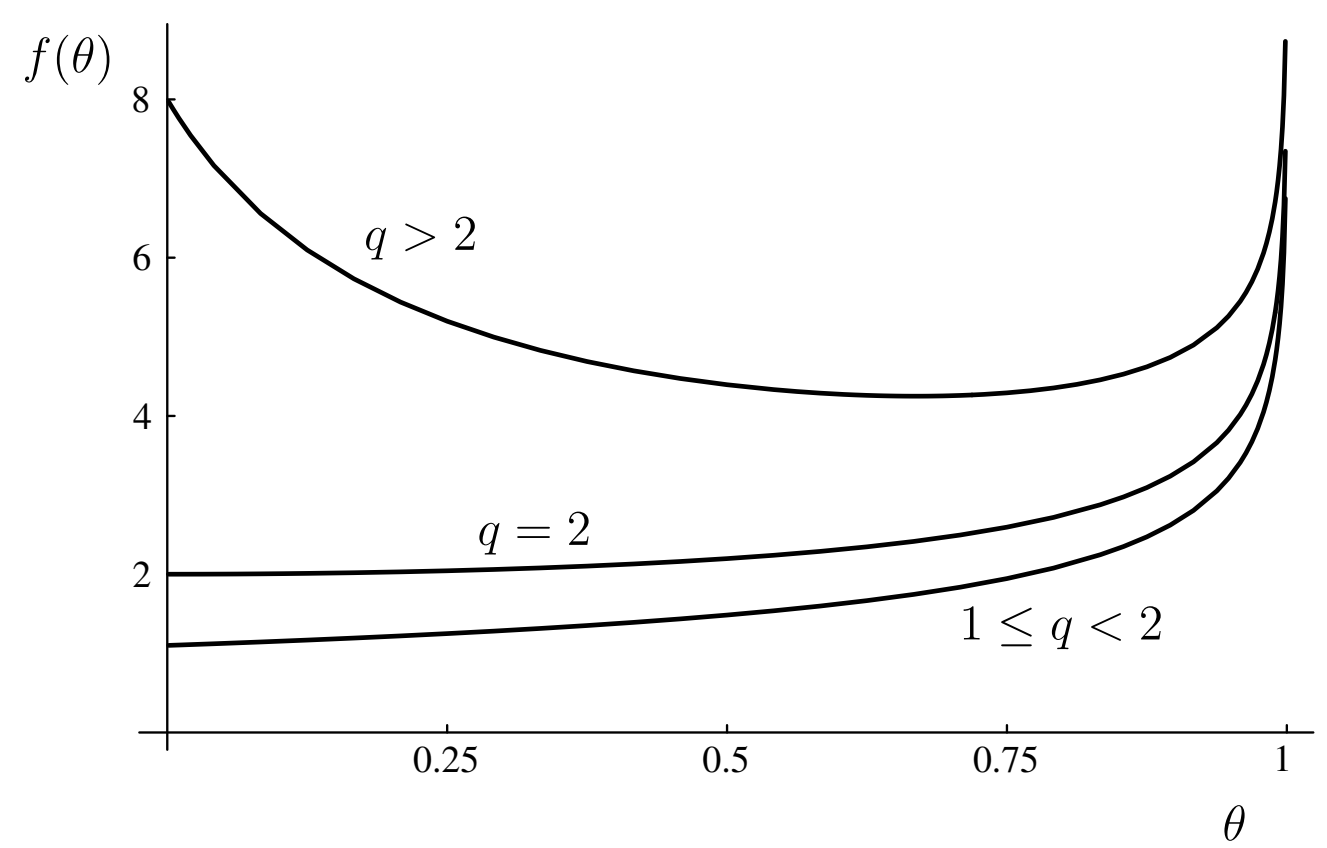

Figure 1. Sketches of the function $f(\theta)$ in the three cases $1 \leq q<2, q=2$, and $q>2$. The respective values of $q$ can be read off from the $y$-axis, since $f(0+)=q$. Note that $f$ is strictly increasing if and only if $q \leq 2$, and $f^{\prime}(0)=0$ when $q=2$. Recall that the positive roots of (2.3) are obtained by intersecting the graph of $f$ by the horizontal line $f(\theta)=\lambda$.

implying $f(1-)=\infty$. Applying Taylor's theorem about $\theta=0$, we find that

$$
f(\theta)=\frac{1}{\theta}\left\{(q-1) \theta-\frac{1}{2}(q-1)^{2} \theta^{2}+\theta+\frac{1}{2} \theta^{2}+\mathrm{O}\left(\theta^{3}\right)\right\}=q+\frac{1}{2} q(2-q) \theta+\mathrm{O}\left(\theta^{2}\right),
$$

whence $f(0+)=q$ and $f^{\prime}(0+)=\frac{1}{2} q(2-q)$. These facts imply assertions (a) and (b) of the lemma.

In Figure 1, we plot $f$ against $\theta$ in the three cases $1 \leq q<2, q=2$, and $q>2$. Since $0<\theta<1$ is a root of (2.3) if and only if $f(\theta)=\lambda$, Lemma 2.4 has the following immediate consequence.

Lemma 2.5. The non-negative roots of equation (2.3) are given as follows, in addition to the root $\theta=0$.

(a) Suppose $0<q \leq 2$.

(i) If $0<\lambda \leq \lambda_{\mathrm{c}}(q)=q$, there is a unique root $\theta=0$.

(ii) If $q<\lambda$, there is a unique positive root $\theta_{\max }(\lambda)$, which satisfies $\theta_{\max }(q+)=$ 0 .

(b) Suppose $q>2$, and let $\lambda_{\min }=f\left(\theta_{\min }\right)$ where $\theta_{\min }$ is given in Lemma 2.4.

(i) If $0<\lambda<\lambda_{\min }$, there is a unique root $\theta=0$.

(ii) If $\lambda=\lambda_{\min }$, then $\theta_{\min }$ is the unique positive root.

(iii) If $\lambda_{\min }<\lambda<q$, there are exactly two positive roots, $\theta_{1}(\lambda)$ and $\theta_{\max }(\lambda)$.

(iv) If $\lambda \geq q$, there is a unique positive root $\theta_{\max }(\lambda)$. 
We shall see later that $\lambda_{\min }<\lambda_{\mathrm{c}}(q)<q$ when $q>2$, and that the function $\theta(\lambda)$ of Theorems 2.1-2.3 satisfies

$$
\theta\left(\lambda_{\mathrm{c}}(q)\right)= \begin{cases}0 & \text { if } q \leq 2 \\ \frac{q-2}{q-1} & \text { if } q>2\end{cases}
$$

Furthermore, we shall obtain the following asymptotic result for the 'free energy'.

Theorem 2.6. If $q>0$ and $\lambda>0$, then

$$
\frac{1}{n} \log Z(n, \lambda / n, q) \rightarrow \phi(\lambda) \quad \text { as } n \rightarrow \infty
$$

where the 'free energy' $\phi(\lambda)=\phi(\lambda, q)$ is given by

$$
\phi(\lambda)=\frac{g(\theta(\lambda))}{2 q}-\frac{q-1}{2 q} \lambda+\log q
$$

and $g(\theta)$ is defined in (6.17).

The proofs of the main theorems (2.1-2.3 and 2.6) are given in Sections 3-6 for $q>1$, and Section 9 for $0<q<1$.

\section{The fundamental lemma}

The following technique is fundamental to the argument of this paper, and is used to reduce the study of $G(n, p, q)$ to the study of the usual random graph $G(n, p, 1)$.

Let $0 \leq r \leq 1$ be fixed. Given a random graph $G(n, p, q)$, colour each component either red, with probability $r$, or green, with probability $1-r$; different components are coloured independently of one other. Thus, if $C_{1}, \ldots, C_{k}$ are the components, then the probability that $C_{i_{1}}, \ldots, C_{i_{l}}$ are red and the other $k-l$ components are green is $r^{l}(1-r)^{k-l}$. The union of the red components is the red subgraph of $G(n, p, q)$, and the green components form the green subgraph. Let $R$ be the set of red vertices, i.e., the vertex-set of the red subgraph.

Lemma 3.1. Let $V_{1} \subseteq V=\{1,2, \ldots, n\}$, with $\left|V_{1}\right|=n_{1}$. Conditional on $R=V_{1}$, the red subgraph of $G(n, p, q)$ is distributed as $G\left(n_{1}, p, r q\right)$ and the green subgraph as $G\left(n-n_{1}, p,(1-r) q\right)$; furthermore, the red subgraph is conditionally independent of the green subgraph.

Proof. Set $V_{2}=V \backslash V_{1}, n_{2}=\left|V_{2}\right|=n-n_{1}$, and let $E_{i} \subseteq V_{i}^{(2)}$ for $i=1,2$. As in (1.1), let us write $c(U, F)$ for the number of components of the graph with vertex set $U$ and edge set $F$. Then $c\left(V, E_{1} \cup E_{2}\right)=c\left(V_{1}, E_{1}\right)+c\left(V_{2}, E_{2}\right)$, whence the probability that the red graph is $\left(V_{1}, E_{1}\right)$ and the green graph is $\left(V_{2}, E_{2}\right)$ satisfies

$$
\begin{aligned}
\left\{\frac{p^{\left|E_{1} \cup E_{2}\right|}(1-p)^{\left(\begin{array}{c}
n \\
2
\end{array}\right)-\left|E_{1} \cup E_{2}\right|} q^{c\left(V, E_{1} \cup E_{2}\right)}}{Z(n, p, q)}\right\} r^{c\left(V_{1}, E_{1}\right)}(1-r)^{c\left(V_{2}, E_{2}\right)} \\
=C\left(n, p, q, n_{1}\right) \mathrm{P}\left(E_{1} ; V_{1}, p, r q\right) \mathrm{P}\left(E_{2} ; V_{2}, p,(1-r) q\right) .
\end{aligned}
$$


for some positive real $C\left(n, p, q, n_{1}\right)$ depending only on $n, p, q$, and $n_{1}$. Hence, conditional on $R=V_{1}$ and the green subgraph being $\left(V_{2}, E_{2}\right)$, the probability that the red subgraph is $\left(V_{1}, E_{1}\right)$ is precisely $P\left(E_{1} ; V_{1}, p, r q\right)$.

In what follows, we shall write $N$, rather than $n_{1}$, for the number of red vertices. Thus $N$ is a random variable and $G(N, p, q r)$ is a random graph on a random number of vertices.

If $q \geq 1$ and $r=q^{-1}$, then the red subgraph is distributed as $G(N, p, 1)$. By studying the distribution of $N$ and using known facts about $G(N, p, 1)$, one may deduce much about the structure of $G(n, p, q)$. Similarly, in order to study the random-cluster model with $q<1$, one applies Lemma 3.1 to $G(n, p, 1)$ with $r=q$, obtaining that the red subgraph is distributed as $G(N, p, q)$; by using known facts about $G(n, p, 1)$, together with some distributional properties of $N$, we may obtain results for $G(m, p, q)$ when $m$ is large.

As an example of the lemma in action, we present a corollary which will be of use later. The quantity $n^{3 / 4}$ is not optimal here, and may be replaced by $n^{2 / 3} \log n$.

Lemma 3.2. Let $q \geq 1$. For any sequence $p=p(n)$, a.e. $G(n, p, q)$ has at most one component having order at least $n^{3 / 4}$.

Proof. Let $L_{n, p, q}$ be the number of components of $G(n, p, q)$ having order at least $n^{3 / 4}$. Suppose $L_{n, p, q} \geq 2$, and pick two of these in some arbitrary way. With probability $r^{2}$ both of these are coloured red. Setting $r=q^{-1}$, we find that

$$
\begin{aligned}
r^{2} \mathrm{P}\left(L_{n, p, q} \geq 2\right) & \leq \sum_{n^{3 / 4} \leq m \leq n} \mathrm{P}\left(L_{m, p, 1} \geq 2\right) \mathrm{P}(|R|=m) \\
& \leq \max _{n^{3 / 4} \leq m \leq n} \mathrm{P}\left(L_{m, p, 1} \geq 2\right) \rightarrow 0 \quad \text { as } n \rightarrow \infty,
\end{aligned}
$$

which follows from Bollobás (1985), Thm VI.9. We note the abuse of notation in writing $L_{m, p, 1}$ without specifying the sample space.

\section{The order of the largest component}

Until further notice we assume that $q \geq 1$. Let $\Theta_{n} n$ be the number of vertices in the largest component of $G(n, p, q)$ where $p=\lambda / n$ and $q \geq 1$; note that $0<\Theta_{n} \leq 1$. If two or more 'largest components' exist, then we pick one of these at random. All other components are called 'small', and it is a consequence of Lemma 3.2 that all small components have orders less than $n^{3 / 4}$ (for a.e. $G(n, \lambda / n, q)$ ).

Consider the colouring scheme of Section 3, with $r=q^{-1}$, and suppose that $G(n, \lambda / n, q)$ has components of order $\Theta_{n} n, \nu_{2}, \nu_{3}, \ldots, \nu_{c}$ where $c$ is the total number of components and we shall assume that $\nu_{i} \leq n^{3 / 4}$ for $i \geq 2$. The number of red vertices in the small components has conditional expectation

$$
\sum_{i=2}^{c} \nu_{i} r=r\left(1-\Theta_{n}\right) n
$$


and variance given by

$$
\sum_{i=2}^{c} \nu_{i}^{2} r(1-r) \leq \sum_{i=2}^{c} \nu_{i}^{2} \leq n \max _{i \geq 2} \nu_{i} \leq n^{7 / 4} .
$$

Hence there are $r\left(1-\Theta_{n}\right) n+\mathrm{O}_{\mathrm{p}}\left(n^{7 / 8}\right)=r\left(1-\Theta_{n}\right) n+\mathrm{o}_{\mathrm{p}}(n)$ red vertices in the small components.

Since the largest component may or may not be coloured red, there are two possibilities for the red graph:

(i) with probability $r$, it has $\Theta_{n} n+r\left(1-\Theta_{n}\right) n+\mathrm{o}_{\mathrm{p}}(n)=\left\{r+(1-r) \Theta_{n}\right\} n+\mathrm{o}_{\mathrm{p}}(n)$ vertices, of which $\Theta_{n} n$ belong to the largest component,

(ii) with probability $(1-r)$, it has $r\left(1-\Theta_{n}\right) n+\mathrm{o}_{\mathrm{p}}(n)$ vertices and the largest component has order less than $n^{3 / 4}$.

In the first case, the red graph is distributed as a supercritical $G\left(n^{\prime}, \lambda^{\prime} / n^{\prime}\right)$ graph, and in the second case as a subcritical $G\left(n^{\prime \prime}, \lambda^{\prime \prime} / n^{\prime \prime}\right)$ graph; here $n^{\prime}$ and $n^{\prime \prime}$ are random, and (with probability tending to 1 ) $\lambda^{\prime}=n^{\prime} p>1>\lambda^{\prime \prime}=n^{\prime \prime} p$. This leads to the next lemma.

Lemma 4.1. If $\lambda>q \geq 1$, then there exists $\theta_{0}$ (>0) such that $\Theta_{n} \geq \theta_{0}$ for a.e. $G(n, \lambda / n, q)$.

Proof. For $q=1$, the assertion is well known; see for example Bollobás (1985), Thm VI.11. Hence assume $q>1$ and thus $r<1$.

Let $\theta_{0}=(\lambda-q) /(2 \lambda), \pi_{n}=\mathrm{P}\left(\Theta_{n}<\theta_{0}\right)$, and $\epsilon>0$. By considering the event that the largest component is not coloured red, we see that, with probability at least $(1-r) \pi_{n}+\mathrm{o}(1)$, the number $N$ of red vertices satisfies $N \geq r\left(1-\theta_{0}\right) n-\epsilon n$, and there are no red components of order at least $n^{3 / 4}$. When this happens,

$$
N p \geq \lambda\left\{r\left(1-\theta_{0}\right)-\epsilon\right\}=\frac{1}{2}+\frac{\lambda}{2 q}-\epsilon \lambda>1
$$

if $\epsilon$ is sufficiently small; pick $\epsilon$ accordingly. Conditional on $N$, a.e. $G(N, p)$ has a component of order at least $\delta N(\geq \delta n / \lambda$ by (4.1)) for some $\delta(>0)$. Therefore $(1-r) \pi_{n} \rightarrow 0$ as $n \rightarrow \infty$.

Lemma 4.2. If $q \geq 1$, then for any sequence $\lambda=\lambda_{n}$, we have that

$$
e^{-\lambda_{n} \Theta_{n}}-\frac{1-\Theta_{n}}{1+(q-1) \Theta_{n}} \stackrel{\mathrm{P}}{\rightarrow} 0 \quad \text { as } n \rightarrow \infty .
$$

Proof. For $q=1$ and constant $\lambda=\lambda_{n}$, this follows from the well known fact that $\Theta_{n} \stackrel{\mathrm{P}}{\rightarrow} \theta$ where $e^{-\lambda \theta}=1-\theta$ (see Bollobás (1985), Thm VI.11 and the remark after Thm V.7); the case of varying $\lambda_{n}$ is not hard to deduce by standard arguments using the conclusion for constant $\lambda$ (this is done by looking down subsequences along which $\lambda_{n}$ converges, with limit lying in $\left.[0, \infty]\right)$. We may express this as

$$
e^{-p_{n} \Theta_{n} n}+\frac{\Theta_{n} n}{n}-1 \stackrel{\mathrm{P}}{\rightarrow} 0 \quad \text { when } q=1,
$$


for the model $G\left(n, p_{n}, 1\right)$ and any sequence $\left(p_{n}\right)$. Applying this to the red subgraph, on the event that it contains the largest component of $G(n, \lambda / n, q)$, we obtain for general $q(\geq 1)$ that

$$
e^{-\lambda \Theta_{n}}+\frac{\Theta_{n}}{r+(1-r) \Theta_{n}}-1=e^{-p \Theta_{n} n}+\frac{\Theta_{n} n}{N}-1+\mathrm{o}_{\mathrm{p}}(1) \stackrel{\mathrm{P}}{\longrightarrow} 0
$$

as $n \rightarrow \infty$, where $N$ is the number of red vertices. The result follows.

Combining these lemmas we arrive at the following theorem.

\section{Theorem 4.3.}

(a) If $1 \leq q \leq 2$ and $\lambda \leq q$, or if $q>2$ and $\lambda<\lambda_{\min }$ where $\lambda_{\min }$ is given in Lemma 2.5, then $\Theta_{n} \stackrel{\mathrm{P}}{\rightarrow} 0$ as $n \rightarrow \infty$.

(b) If $q \geq 1$ and $\lambda>q$, then $\Theta_{n} \stackrel{\mathrm{P}}{\rightarrow} \theta(\lambda)$ where $\theta(\lambda)$ is the unique (strictly) positive solution of (2.3).

This goes some way towards proving Theorems 2.1 and 2.2. Overlooking for the moment the more detailed asymptotical claims of those theorems, we note that the major remaining gap is when $q>2$ and $\lambda_{\min } \leq \lambda \leq q$. In this case, it follows from Lemma 4.2 that $\Theta_{n}$ is approximately equal to one of the three roots of (2.3) (including the trivial root $\theta=0$ ); only after the analysis of the next two sections shall we see which root is the correct one for a given value of $\lambda$.

Proof. The function

$$
\phi(\theta)=e^{-\lambda \theta}-\frac{1-\theta}{1+(q-1) \theta}
$$

is continuous on $[0,1]$; the set $Z$ of zeros of $g$ is described in Lemma 2.5. Since $\phi\left(\Theta_{n}\right) \stackrel{\mathrm{P}}{\longrightarrow} 0$, by Lemma 4.2 , it follows that, for all $\epsilon>0$,

$$
\mathrm{P}\left(\Theta_{n} \in Z+(-\epsilon, \epsilon)\right) \rightarrow 1 \quad \text { as } n \rightarrow \infty
$$

Under the assumption of (a), $Z$ contains the singleton 0 , and the claim follows. Under (b), $Z$ contains exactly one strictly positive number $\theta(\lambda)$, and the claim follows by Lemma 4.1 .

\section{The number of edges}

Given $G(n, p, q)$, pick one of the largest components at random, and write $\Xi_{n} n$ for the number of edges in this component. Also, write $\Psi_{n} n$ for the number of edges of the entire graph $G(n, p, q)$. Assume $q>1$. Arguing as in Sections 3 and 4 with $r=q^{-1}$, we see that a.e. $G(n, p, q)$ has at most $n^{3 / 4}$ edges in each small component (a 'small' component is any component except the largest, picked above). [For this we need the corresponding result for $q=1$, which easily follows from the corresponding result for the number of vertices used above, and results on the components with more edges than vertices given in Bollobás (1984), Janson 
(1993), or Janson, Knuth, Łuczak, and Pittel (1993).] Furthermore the number of red edges in the small components is $r\left(\Psi_{n}-\Xi_{n}\right) n+\mathrm{o}_{\mathrm{p}}(n)$. Hence the red subgraph has either

(i) (with probability $r$ ) $\left\{\Theta_{n}+r\left(1-\Theta_{n}\right)\right\} n+o_{p}(n)$ vertices and $\left\{\Xi_{n}+r\left(\Psi_{n}-\right.\right.$ $\left.\left.\Xi_{n}\right)\right\} n+\mathrm{o}_{\mathrm{p}}(n)$ edges, or

(ii) (otherwise) $r\left(1-\Theta_{n}\right) n+\mathrm{o}_{\mathrm{p}}(n)$ vertices and $r\left(\Psi_{n}-\Xi_{n}\right) n+\mathrm{o}_{\mathrm{p}}(n)$ edges.

Assume that $p=\mathrm{O}\left(n^{-1}\right)$. Since a.e. $G(N, p)$ graph has $\left(\begin{array}{c}N \\ 2\end{array}\right) p+\mathrm{O}_{\mathrm{p}}\left(N p^{1 / 2}\right)=$ $\frac{1}{2} N^{2} p+\mathrm{o}_{\mathrm{p}}(N)$ edges, the following two equations follow from the two cases above,

$$
\begin{gathered}
\left\{\Xi_{n}+r\left(\Psi_{n}-\Xi_{n}\right)\right\} n=\frac{1}{2}\left\{\Theta_{n}+r\left(1-\Theta_{n}\right)\right\}^{2} n^{2} p+\mathrm{o}_{\mathrm{p}}(n), \\
r\left(\Psi_{n}-\Xi_{n}\right) n=\frac{1}{2}\left\{r\left(1-\Theta_{n}\right)\right\}^{2} n^{2} p+\mathrm{o}_{\mathrm{p}}(n),
\end{gathered}
$$

yielding when $p=\lambda / n$ that

$$
\begin{aligned}
\Xi_{n}+r\left(\Psi_{n}-\Xi_{n}\right) & =\frac{1}{2} \lambda\left\{\Theta_{n}+r\left(1-\Theta_{n}\right)\right\}^{2}+\mathrm{o}_{\mathrm{p}}(1), \\
r\left(\Psi_{n}-\Xi_{n}\right) & =\frac{1}{2} \lambda\left\{r\left(1-\Theta_{n}\right)\right\}^{2}+\mathrm{o}_{\mathrm{p}}(1) .
\end{aligned}
$$

We solve for $\Xi_{n}$ and $\Psi_{n}$, and pass to the limit as $n \rightarrow \infty$, to obtain the next theorem.

Theorem 5.1. If $q>1$ and $\lambda>0$, then, as $n \rightarrow \infty$,

$$
\begin{array}{r}
\Psi_{n}-\frac{\lambda}{2 q}\left\{1+(q-1) \Theta_{n}^{2}\right\} \stackrel{\mathrm{P}}{\longrightarrow} 0, \\
\Xi_{n}-\frac{\lambda}{q} \Theta_{n}\left\{1+\left(\frac{1}{2} q-1\right) \Theta_{n}\right\} \stackrel{\mathrm{P}}{\longrightarrow} 0 .
\end{array}
$$

Whereas we proved this theorem under the assumption that $q>1$, its conclusions are valid for $q=1$ also (a consequence of Bollobás (1985), Thms VI.11 and VI.12). Note that the proof used information about the number of edges in a $G(n, p)$ graph but no information about the number of edges in its largest component.

\section{Proofs of main results}

In this section we combine the results derived so far with a new argument in order to prove Theorems 2.1-2.3 for $q \geq 1$.

We note first that the results of the theorems are well known when $q=1$ (see Bollobás (1985), Chaps V and VI, and Łuczak, Pittel, and Wierman (1994)), and henceforth we assume that $q>1$. We define the acyclic part of a graph to be the union of all components that are trees, and the cyclic part to be the union of the remaining components. We call a graph cyclic if its acyclic part is empty. We being by showing that the cyclic part of a.e. $G(n, \lambda / n, q)$ consists principally of the largest component only (when this component is cyclic).

Lemma 6.1. The numbers of vertices and edges in the small cyclic components of $G(n, \lambda / n, q)$ are $\mathrm{o}_{\mathrm{p}}(n)$.

Proof. Let $k$ be an integer satisfying $k \geq q$. In the colouring scheme of Section 3 with $r=q^{-1}$, we introduce the refinement that each component is coloured dark red 
with probability $k^{-1}$ and light red with probability $r-k^{-1}$. Let $M$ be the number of edges in the small cyclic components of $G(n, \lambda / n, q)$. By a symmetry argument, with probability at least $k^{-1}$, at least $M / k$ of these edges are coloured dark red. [Let $M_{i}$ be the number of such edges coloured $\chi_{i}$, when each component is coloured by a random colour from the set $\left\{\chi_{1}, \chi_{2}, \ldots, \chi_{k}\right\}$, each having equal probability. If $\mathrm{P}\left(M_{i} \geq M / k\right)<k^{-1}$, then $\mathrm{P}\left(M_{i} \geq M / k\right.$ for some $\left.i\right)<1$, which is a contradiction since $\sum_{i=1}^{k} M_{i}=M$.] Therefore, with probability at least $r / k$, the red subgraph contains the largest component together with small cyclic components having at least $M / k$ edges. The result follows from the known case $q=1$; see Bollobás (1984) and Bollobás (1985), Thm VI.11.

Let $\widetilde{P}(m, j, k, l ; n, p, q)$ be the sum of all $\widetilde{P}(E ; n, p, q)$ over edge-sets $E$ that define a graph with $|E|=m$ edges and a cyclic part with $j$ components, $k$ vertices, and $l$ edges. Since such graphs have an acyclic part with $n-k$ vertices and $m-l$ edges, and therefore $n-k-m+l$ components, we obtain

$$
\widetilde{P}(m, j, k, l ; n, p, q)=\left(\begin{array}{l}
n \\
k
\end{array}\right) c(j, k, l) f(n-k, m-l) p^{m}(1-p)^{\left(\begin{array}{l}
n \\
2
\end{array}\right)-m} q^{n-k-m+l+j}
$$

where $c(j, k, l)$ is the number of cyclic graphs with $j$ components, $k$ labelled vertices, and $l$ edges, and similarly $f(n, m)$ is the number of forests with $n$ labelled vertices and $m$ edges.

Let us now assume that $n \rightarrow \infty$, that $\lambda=n p>0$ and $q \geq 1$ are fixed, and that

$$
m / n \rightarrow \psi, k / n \rightarrow \theta, l / n \rightarrow \xi, \text { and } j / n \rightarrow 0,
$$

where $\theta(\geq 0)$ satisfies $(2.3)$, and

$$
\begin{aligned}
\xi & =\frac{\lambda}{q} \theta\left\{1+\left(\frac{1}{2} q-1\right) \theta\right\}, \\
\psi & =\xi+\frac{\lambda}{2 q}(1-\theta)^{2} ;
\end{aligned}
$$

see (5.5) and (5.6). If $\lambda>q$, we also assume that $\theta>0$, cf. Lemma 4.1 and Theorem $4.3(\mathrm{~b})$.

Since $f(n, m) \leq\left(\begin{array}{c}n \\ 2 \\ m\end{array}\right)$, the total number of graphs with $m$ edges on $n$ vertices, we have that

$$
\begin{aligned}
& \widetilde{P}(m, j, k, l ; n, p, q) \leq\left(\begin{array}{c}
n \\
k
\end{array}\right) c(j, k, l)\left(\begin{array}{c}
\left(\begin{array}{c}
n-k \\
2
\end{array}\right) \\
m-l
\end{array}\right) p^{m}(1-p)^{\left(\begin{array}{c}
n \\
2
\end{array}\right)-m} q^{n-k-m+l+j} \\
& =\left(\begin{array}{l}
n \\
k
\end{array}\right) c(j, k, l)\left(\begin{array}{c}
n-k \\
2
\end{array}\right)^{m-l}\left(\frac{e}{m-l}\right)^{m-l} p^{m} q^{n-k-m+l} \exp \left(-\frac{1}{2} \lambda n+o(n)\right) \\
& =c(j, k, l)\left(\begin{array}{l}
n \\
k
\end{array}\right)\left(\frac{(n-k)^{2} e \lambda}{2(m-l) n}\right)^{m-l} p^{l} q^{n-k-m+l} \exp \left(-\frac{1}{2} \lambda n+o(n)\right) \\
& =p^{l} c(j, k, l)\left(\begin{array}{l}
n \\
k
\end{array}\right)\left(\frac{(1-\theta)^{2} \lambda}{2(\psi-\xi)}\right)^{m-l} q^{n-k-m+l} \exp \left(m-l-\frac{1}{2} \lambda n+o(n)\right) \\
& =p^{l} e^{-l} c(j, k, l)\left(\begin{array}{l}
n \\
k
\end{array}\right) q^{n-k} \exp \left(m-\frac{1}{2} \lambda n+o(n)\right),
\end{aligned}
$$


where we used (6.4) in the last step.

We shall be interested only in values of $\lambda$ and roots $\theta$ of (2.3) satisfying

$$
\text { either } \theta>0 \text {, or } \theta=0 \text { and } \lambda \leq q \text {. }
$$

We claim that, under these assumptions, (6.5) is an equality:

$$
\widetilde{P}(m, j, k, l ; n, p, q)=p^{l} e^{-l} c(j, k, l)\left(\begin{array}{l}
n \\
k
\end{array}\right) q^{n-k} \exp \left(m-\frac{1}{2} \lambda n+\mathrm{o}(n)\right) .
$$

To see this when either $\theta>0$, or $\theta=0$ and $\lambda<q$, set $n_{0}=n-k$ and $m_{0}=m-l$, and observe that

$$
\frac{m_{0}}{n_{0}}=\frac{m-l}{n-k} \rightarrow \frac{\psi-\xi}{1-\theta}=\frac{\lambda}{2 q}(1-\theta)<\frac{1}{2},
$$

where we have used the fact that, by (2.3),

$$
\lambda \theta<e^{\lambda \theta}-1=\frac{q \theta}{1-\theta} \quad \text { when } \theta>0 .
$$

Hence in this case, the 'fixed edge-number' random graph $G\left(n_{0}, m_{0}\right)$ has average vertex degree not exceeding $1-\epsilon$ for some positive constant $\epsilon$ independent of $n, m, k, l$. Therefore there exists a positive constant $\delta$ such that

$$
\mathrm{P}\left(G\left(n_{0}, m_{0}\right) \text { is a forest }\right)>\delta,
$$

and hence

$$
\left.f\left(n_{0}, m_{0}\right)>\delta\left(\begin{array}{c}
n_{0} \\
2
\end{array}\right)\right) ;
$$

this implies (6.7), via (6.1) and (6.5). When $\theta=0$ and $\lambda=q$, we have that $m_{0} / n_{0} \rightarrow \psi=\frac{1}{2}$, and hence

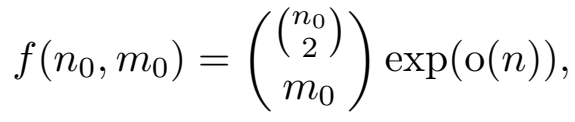

implying (6.7). To see (6.8), note that with $0<\epsilon<\frac{1}{4}$ and $s \asymp \epsilon n$ we have for large $n$, rather crudely by counting only forests where vertex 1 is an endpoint of an isolated path of length $s-1$, that

$$
\begin{aligned}
f\left(n_{0}, m_{0}\right) & \geq\left(n_{0}-1\right)_{s-1} f\left(n_{0}-s, m_{0}-s+1\right) \\
& \geq e^{-\epsilon n} n_{0}^{s}\left(\begin{array}{c}
\left(\begin{array}{c}
n_{0}-s \\
2
\end{array}\right) \\
m_{0}-s+1
\end{array}\right) \geq e^{2 \log (1-\epsilon) n}\left(\begin{array}{c}
\left(\begin{array}{c}
n_{0} \\
2
\end{array}\right) \\
m_{0}
\end{array}\right) .
\end{aligned}
$$

Alternatively, we may use the detailed asymptotics for $f(r, s)$ of Britikov (1988). 
We estimate $c(j, k, l)$ next. Suppose first that $\theta=0$. Then $c(j, k, l)$ is no greater than the total number of graphs with $k$ vertices and $l$ edges, i.e.,

$$
\begin{aligned}
p^{l} e^{-l} c(j, k, l) & \leq\left(\frac{p}{e}\right)^{l}\left(\frac{k^{2}}{2}\right)^{l} \frac{1}{l !} \leq\left(\frac{\lambda n}{l}\right)^{l} \\
& =\exp \left\{l\left(\log \lambda-\log \frac{l}{n}\right)\right\}=\exp (\mathrm{o}(n)) .
\end{aligned}
$$

Equality holds here for some suitable triple $j, k, l$ : just set $j=k=l=0$, for which $p^{l} e^{-l} c(j, k, l)=1$. It is easily checked that $\left(\begin{array}{l}n \\ k\end{array}\right)=\exp (\mathrm{o}(n))$ when $\theta=0$, and therefore

$$
p^{l} e^{-l} c(j, k, l) \leq\left(\begin{array}{l}
n \\
k
\end{array}\right)^{-1} \exp (\mathrm{o}(n))
$$

with equality for some suitable $j, k, l$.

Our estimate of $c(j, k, l)$ when $\theta>0$ uses the fact that $\widetilde{P}(\cdot ; n, p, q)$ is a probability measure when $q=1$. Suppose $\theta>0$, define $n_{1}=n_{1}(\theta)=\lfloor\theta n+r(1-\theta) n\rfloor$ where $r=q^{-1}$ as usual, and set

$$
\begin{aligned}
m_{1} & =l+r(m-l)+\mathrm{o}(n)=\{\xi+r(\psi-\xi)\} n+\mathrm{o}(n), \\
\lambda_{1} & =\{\theta+r(1-\theta)\} \lambda .
\end{aligned}
$$

Then

$$
\begin{aligned}
\frac{m_{1}}{n_{1}} \rightarrow \psi_{1} & =\frac{\xi+r(\psi-\xi)}{\theta+r(1-\theta)}=\frac{1}{2} \lambda_{1}, \\
\frac{k}{n_{1}} \rightarrow \theta_{1} & =\frac{\theta}{\theta+r(1-\theta)}, \\
\frac{l}{n_{1}} \rightarrow \xi_{1} & =\frac{\xi}{\theta+r(1-\theta)}, \quad \frac{j}{n_{1}} \rightarrow 0 ;
\end{aligned}
$$

it is easy to check the analogues of (2.3), (6.3), and (6.4), viz.

$$
e^{-\lambda_{1} \theta_{1}}=1-\theta_{1}, \quad \xi_{1}=\lambda_{1} \theta_{1}\left(1-\frac{1}{2} \theta_{1}\right), \quad \psi_{1}=\xi_{1}+\frac{1}{2} \lambda_{1}\left(1-\theta_{1}\right)^{2} .
$$

Now (6.7) is valid with $q=1$, since $\theta>0$. Hence

$$
\begin{aligned}
1 & \geq \widetilde{P}\left(m_{1}, j, k, l ; n_{1}, p_{1}, 1\right) \\
& =p_{1}^{l} e^{-l} c(j, k, l)\left(\begin{array}{c}
n_{1} \\
k
\end{array}\right) \exp \left(m_{1}-\frac{1}{2} \lambda_{1} n_{1}+\mathrm{o}(n)\right) \\
& =p^{l} e^{-l} c(j, k, l)\left(\begin{array}{c}
n_{1} \\
k
\end{array}\right) \exp (\mathrm{o}(n))
\end{aligned}
$$

by (6.10), where $p_{1}=\lambda_{1} / n_{1}=p\left(1+\mathrm{O}\left(n^{-1}\right)\right)$. Therefore

$$
p^{l} e^{-l} c(j, k, l) \leq\left(\begin{array}{c}
n_{1} \\
k
\end{array}\right)^{-1} \exp (\mathrm{o}(n)) .
$$


We claim that there exist suitable $j, k, l$ such that equality holds in (6.15). To see this, note that $G\left(n_{1}, p_{1}\right)$ has $\left(\begin{array}{c}n_{1} \\ 2\end{array}\right) p_{1}+\mathrm{o}_{\mathrm{p}}\left(n_{1}\right)$ edges, a giant component with $\theta_{1} n_{1}+\mathrm{o}_{\mathrm{p}}\left(n_{1}\right)$ vertices and $\xi_{1} n_{1}+\mathrm{o}_{\mathrm{p}}\left(n_{1}\right)$ edges, $\mathrm{O}_{\mathrm{p}}(1)$ unicyclic components with a total of $\mathrm{O}_{\mathrm{p}}(1)$ vertices and edges, and no other cyclic components (see Bollobás (1985), Thm VI.11). By considering the number of possible combinations of values of $m_{1}, j, k, l$ satisfying the above constraints, we find that there must exist $m_{1}, j, k, l$ such that

$$
\widetilde{P}\left(m_{1}, j, k, l ; n_{1}, p_{1}, 1\right) \geq n^{-4}
$$

for all large $n$. Combining this with (6.14), we deduce that equality holds in (6.15) for some suitable $j, k, l$.

In conclusion, we have that, whatever the root $\theta$ of (2.3) (subject to (6.6)), inequality (6.15) holds with equality for some suitable $j, k, l$, and where $n_{1}=n_{1}(0)$ is interpreted as $n$ (i.e., when $\theta=0$ ). We substitute (6.15) into (6.5) to obtain

$$
\begin{aligned}
\widetilde{P}(m, j, k, l ; n, p, q) \leq\left(\begin{array}{c}
n_{1} \\
k
\end{array}\right)^{-1}\left(\begin{array}{l}
n \\
k
\end{array}\right) q^{n-k} \exp \left(m-\frac{1}{2} \lambda n+\mathrm{o}(n)\right) \\
=\frac{n^{n}}{(n-k)^{n-k}} \frac{\left(n_{1}-k\right)^{n_{1}-k}}{n_{1}^{n_{1}}} q^{n-k} \exp \left(\frac{\lambda}{2 q}\left\{1+(q-1) \theta^{2}\right\} n-\frac{1}{2} \lambda n+\mathrm{o}(n)\right) \\
=\left[\frac{\{r(1-\theta)\}^{r(1-\theta)}}{(1-\theta)^{1-\theta}\{\theta+r(1-\theta)\}^{\theta+r(1-\theta)}} q^{1-\theta} \times\right. \\
\left.\quad \exp \left(\frac{\lambda}{2 q}\left\{1+(q-1) \theta^{2}\right\}-\frac{1}{2} \lambda+\mathrm{o}(1)\right)\right]^{n} \\
=\exp \left(n\left[\frac{g(\theta)}{2 q}-\frac{q-1}{2 q} \lambda+\log q+\mathrm{o}(1)\right]\right),
\end{aligned}
$$

where

$$
g(\theta)=-(q-1)(2-\theta) \log (1-\theta)-\{2+(q-1) \theta\} \log \{1+(q-1) \theta\} .
$$

To obtain the second line of (6.16), we have used (6.3) and (6.4). To pass to the last line, we used the fact that $\theta$ is a root of (2.3), to enable the substitution

$$
\exp \left(\frac{\lambda}{2 q}\left\{1+(q-1) \theta^{2}\right\}\right)=e^{\lambda /(2 q)}\left\{\frac{1+(q-1) \theta}{1-\theta}\right\}^{(q-1) \theta /(2 q)}
$$

We have in addition that equality holds in (6.16) for at least one suitable choice of $j, k, l$. Let $\theta^{*}=\theta^{*}(\lambda)$ be the root of (2.3) which maximizes $g(\theta)$ and which satisfies (6.6) [we shall see that there is a unique such $\theta^{*}$, except when $\lambda=\lambda_{c}(q)$ and $q>2$ ]. By (6.16) and the equality observed above,

$$
\begin{aligned}
Z(n, p, q) & =\sum_{m, j, k, l} \widetilde{P}(m, j, k, l ; n, p, q) \\
& \geq \exp \left\{n\left[\frac{g\left(\theta^{*}\right)}{2 q}-\frac{q-1}{2 q} \lambda+\log q+\mathrm{o}(1)\right]\right\},
\end{aligned}
$$


whence

$$
\liminf _{n \rightarrow \infty}\left\{\frac{1}{n} \log Z(n, p, q)\right\} \geq \frac{g\left(\theta^{*}\right)}{2 q}-\frac{q-1}{2 q} \lambda+\log q
$$

On the other hand, by Lemmas 4.1 and 4.2, there exists a root $\theta$ of (2.3) satisfying (6.6), and a function $\omega(n)$ satisfying $\omega(n) \rightarrow \infty$, such that

$$
\liminf _{n \rightarrow \infty} \mathrm{P}\left(\left|\Theta_{n}-\theta\right|<\omega(n)^{-1}\right)>0 .
$$

For such $\theta$ there exist, by Lemma 6.1 and Theorem 5.1 , sequences $m, j, k, l$ satisfying (6.2)-(6.4) and such that

$$
1 \geq \frac{\widetilde{P}(m, j, k, l ; n, p, q)}{Z(n, p, q)} \geq n^{-4}
$$

for all large $n$ (this is shown by considering the number of possible combinations of $m, j, k, l$ satisfying (6.2)-(6.4) and the above-mentioned results). By (6.16), this yields

$$
\limsup _{n \rightarrow \infty}\left\{\frac{1}{n} \log Z(n, p, q)\right\} \leq \frac{g(\theta)}{2 q}-\frac{q-1}{2 q} \lambda+\log q
$$

which, by (6.19), implies $g(\theta) \geq g\left(\theta^{*}\right)$, and therefore $\theta=\theta^{*}$. Combining (6.19) and (6.21), we obtain Theorem 2.6. Furthermore, $\theta^{*}$ is the only root of (2.3) satisfying (6.6) such that (6.20) holds for some $\omega(n)$. Therefore

$$
\Theta_{n} \stackrel{\mathrm{P}}{\rightarrow} \theta^{*} \quad \text { as } n \rightarrow \infty .
$$

Next we calculate $\theta^{*}(\lambda)$. As in Theorem 4.3 , when $1 \leq q \leq 2, \theta^{*}(\lambda)$ is the largest non-negative root of (2.3). Assume that $q>2$. By straightforward computation,

$$
\begin{gathered}
g(0)=g\left(\frac{q-2}{q-1}\right)=0, \quad g^{\prime}(0)=0, \\
g^{\prime \prime}(\theta)=-\frac{q(q-1)\{q-2-2(q-1) \theta\} \theta}{(1-\theta)^{2}\{1+(q-1) \theta\}^{2}} .
\end{gathered}
$$

Therefore $g^{\prime \prime}(\theta)$ has a unique zero in $(0,1)$, at $\theta=\frac{1}{2}(q-2) /(q-1)$; at this point, $g^{\prime}(\theta)$ has a negative minimum. It follows that $g(\theta)<0$ on $\left(0, \theta_{0}\right)$, and $g(\theta)>0$ on $\left(\theta_{0}, 1\right)$ where $\theta_{0}=(q-2) /(q-1)$.

Substituting $\theta_{0}$ into $(2.3)$, we find that $\theta_{0}$ satisfies $(2.3)$ if

$$
\lambda=\lambda_{\mathrm{c}}(q)=2\left(\frac{q-1}{q-2}\right) \log (q-1),
$$

and, for this value of $\lambda$, the three roots of (2.3) are $0, \frac{1}{2} \theta_{0}, \theta_{0}$. It follows that $\lambda_{\min }<\lambda_{\mathrm{c}}(q)<q$, and that $\theta^{*}=0$ for $\lambda<\lambda_{\mathrm{c}}(q)$, and $\theta^{*}=\theta_{\max }(\lambda)$ for $\lambda>\lambda_{\mathrm{c}}(q)$. Finally, when $\lambda=\lambda_{\mathrm{c}}(q)$, both $\theta=0$ and $\theta=\theta_{\max }(\lambda)$ give the same value of $g(\theta)$ 
(namely 0), while the third root yields a smaller value. Hence we can in this case conclude only that

$$
\mathrm{P}\left(\Theta_{n}<\omega(n)^{-1} \text { or }\left|\Theta_{n}-\theta_{\max }\left(\lambda_{\mathrm{c}}(q)\right)\right|<\omega(n)^{-1}\right) \rightarrow 1
$$

for some $\omega(n)$ satisfying $\omega(n) \rightarrow \infty$.

This completes the proof of the assertions concerning the order of the largest component. The claims concerning the number of edges in $G(n, p, q)$ and in the giant component follow by Theorem 5.1. We omit explicit proofs of the remaining assertions about the structure of $G(n, p, q)$; as in Lemma 6.1, these follow easily by the colouring argument from known facts for $G(n, p)$; see Bollobás (1985) and Łuczak, Pittel, and Wierman (1994).

\section{The nature of the singularity}

Let $q(\geq 1)$ be fixed, and consider the functions $\theta(\lambda)$ and

$$
\psi(\lambda)=\frac{\lambda}{2 q}\left\{1+(q-1) \theta(\lambda)^{2}\right\}, \quad \xi(\lambda)=\frac{\lambda}{q}\left\{\theta(\lambda)+\left(\frac{1}{2} q-1\right) \theta(\lambda)^{2}\right\},
$$

describing the order of the giant component, and the numbers of edges in the graph and in its giant component, respectively. All three functions are nondecreasing on $(0, \infty)$. Actually $\psi$ is strictly increasing, while $\theta(\lambda)$ and $\xi(\lambda)$ equal 0 for $\lambda<\lambda_{c}$ and are strictly increasing on $\left[\lambda_{c}, \infty\right)$.

A fourth function of interest is the free energy $\phi(\lambda)$ given in Theorem 2.6. All these four functions are real analytic on $(0, \infty)$ except at $\lambda_{\mathrm{c}}$. At the singularity $\lambda_{\mathrm{c}}$, the following is valid, as may be verified with ease.

(a) Assume $q<2$. Then $\theta, \psi, \xi$, and $\phi$ are continuous at $\lambda_{\mathrm{c}}(q)=q$. The functions $\theta$ and $\xi$ have discontinuous first derivatives at $\lambda_{c}$; in fact

$$
\theta^{\prime}\left(\lambda_{\mathrm{c}}-\right)=\xi^{\prime}\left(\lambda_{\mathrm{c}}-\right)=0, \quad \theta^{\prime}\left(\lambda_{\mathrm{c}}+\right)=\xi^{\prime}\left(\lambda_{\mathrm{c}}+\right)=\frac{2}{q(2-q)} .
$$

In particular

$$
\theta(\lambda) \sim \frac{2\left(\lambda-\lambda_{\mathrm{c}}\right)}{q(2-q)} \quad \text { as } \lambda \downarrow \lambda_{\mathrm{c}}
$$

Similarly, $\psi^{\prime}$ and $\phi^{\prime \prime}$ are continuous, but $\psi^{\prime \prime}$ and $\phi^{\prime \prime \prime}$ have discontinuities at $\lambda_{\mathrm{c}}$, except when $q=1$.

(b) Assume $q=2$. Still $\theta, \psi, \xi$, and $\phi$ are continuous at $\lambda_{\mathrm{c}}$. In this case

$$
\theta(\lambda) \sim \xi(\lambda) \sim\left\{\frac{3}{2}\left(\lambda-\lambda_{\mathrm{c}}\right)\right\}^{1 / 2} \text { as } \lambda \downarrow \lambda_{\mathrm{c}} ;
$$

thus $\theta^{\prime}\left(\lambda_{\mathrm{c}}+\right)=\xi^{\prime}\left(\lambda_{\mathrm{c}}+\right)=\infty$. Also, $\psi^{\prime}$ has a jump at $\lambda_{\mathrm{c}}: \psi^{\prime}\left(\lambda_{\mathrm{c}}-\right)=\frac{1}{4}$, $\psi^{\prime}\left(\lambda_{\mathrm{c}}+\right)=1$. And $\phi^{\prime}$ is continuous, but $\phi^{\prime \prime}$ has a jump at $\lambda_{\mathrm{c}}: \phi^{\prime \prime}\left(\lambda_{\mathrm{c}}-\right)=0$, $\phi^{\prime \prime}\left(\lambda_{\mathrm{c}}+\right)=\frac{3}{8}$. The functions $\psi$ and $\phi$ are real analytic on $\left(0, \lambda_{\mathrm{c}}\right]$ and on $\left[\lambda_{\mathrm{c}}, \infty\right)$.

(c) Assume $q>2$. Then $\theta, \psi$, and $\xi$ have jumps at $\lambda_{\mathrm{c}}$, and it may be verified that $\psi\left(\lambda_{\mathrm{c}}-\right)=\lambda_{\mathrm{c}} /(2 q)<\frac{1}{2}<\psi\left(\lambda_{\mathrm{c}}+\right)$. The free energy $\phi$ is continuous at $\lambda_{\mathrm{c}}$, but its derivative $\phi^{\prime}$ has a jump at $\lambda_{\mathrm{c}}$ :

$$
\phi^{\prime}\left(\lambda_{\mathrm{c}}-\right)=-\frac{q-1}{2 q}, \quad \phi^{\prime}\left(\lambda_{\mathrm{c}}+\right)=-\frac{2 q-3}{2 q(q-1)} .
$$




\section{Large deviations}

Let $C_{n}$ be the number of components of the graph $G(n, \lambda / n, q)$. We show next how the exact calculation of the free energy in Theorem 2.6 may be used to estimate probabilities of the form $\mathrm{P}\left(C_{n} \leq \alpha n\right)$ and $\mathrm{P}\left(C_{n} \geq \beta n\right)$ for given constants $\alpha, \beta$. In the case $q=1$, this gives new information about the probabilities of large deviations of $C_{n}$. (We will use Theorem 2.6 also for $q<1$, even though this case is not proven until the next section.)

As in the language of large-deviation theory (see Dembo and Zeitouni (1993)), we set

$$
\Lambda_{n, \lambda, q}(\nu)=\log \mathrm{E}\left(\exp \left\{\nu C_{n} / n\right\}\right), \quad \nu \in \mathbb{R},
$$

and note that

$$
\Lambda_{n, \lambda, q}(\nu)=\log \left\{\frac{Z\left(n, \lambda / n, q e^{\nu / n}\right)}{Z(n, \lambda / n, q)}\right\},
$$

whence

$$
\frac{1}{n} \Lambda_{n, \lambda, q}(n \nu) \rightarrow \Lambda_{\lambda, q}(\nu)=\phi\left(\lambda, q e^{\nu}\right)-\phi(\lambda, q)
$$

as $n \rightarrow \infty$ (here, $\phi(\lambda, q)$ denotes the free-energy function of Theorem 2.6). We define the Legendre transform of $\Lambda_{\lambda, q}$ by

$$
\Lambda_{\lambda, q}^{*}(x)=\sup _{\nu \in \mathbb{R}}\left\{\nu x-\Lambda_{\lambda, q}(\nu)\right\}, \quad x \in \mathbb{R} .
$$

It may be proved directly (or see Dembo and Zeitouni (1993), Lemma 2.3.9) that $\Lambda_{\lambda, q}$ and $\Lambda_{\lambda, q}^{*}$ are convex functions, and that

$$
\Lambda_{\lambda, q}^{*}(x)=\delta x-\Lambda_{\lambda, q}(\delta) \quad \text { if } \quad \Lambda_{\lambda, q}^{\prime}(\delta)=x .
$$

Since we have an exact formula for $\Lambda_{\lambda, q}$ (see (8.1) and (2.6)), we may compute its derivative whenever it exists (see Theorem 8.2). As a consequence of such computations, we find that

$$
\Lambda_{\lambda, q}^{*}(x) \begin{cases}<\infty & \text { if } 0 \leq x \leq 1 \\ =\infty & \text { otherwise. }\end{cases}
$$

(The cases $x=0,1$ are not used in the sequel, and therefore we omit some extra details required for these cases.)

A large-deviation principle (LDP) may be established for $n^{-1} C_{n}$, in terms of the 'rate function' $\Lambda_{\lambda, q}^{*}$. The details of the LDP depend on the set of points $x$ at which $\Lambda_{\lambda, q}^{*}$ is strictly convex, and we investigate this next. There is a slight complication in this, arising from the discontinuity of the phase transition when $q>2$. The proof of the following theorem is deferred to the end of this section. 
Theorem 8.2. Let $\lambda, q>0$.

(a) The derivative

$$
\kappa(\lambda, q)=q \frac{\partial \phi(\lambda, q)}{\partial q}
$$

exists except when $\lambda=\lambda_{\mathrm{c}}(q)$ and $q>2$, and satisfies

$$
\kappa(\lambda, q)=\lim _{n \rightarrow \infty} \frac{1}{n} \mathrm{E}\left(C_{n}\right) .
$$

Furthermore

$$
\kappa(\lambda, q)=1-\theta(\lambda)-(1-\theta(\lambda))^{2} \frac{\lambda}{2 q}
$$

When $\lambda=\lambda_{\mathrm{c}}(q)$ and $q>2$, the limits

$$
\kappa^{ \pm}(\lambda, q)=q \frac{\partial \phi(\lambda, q \pm)}{\partial q}
$$

exist with $\kappa^{-}(\lambda, q)<\kappa^{+}(\lambda, q)$. Also, $\kappa^{-}(\lambda, q)$ is given by the formula in (8.5), and

$$
\kappa^{+}(\lambda, q)=1-\frac{\lambda}{2 q} .
$$

(b) Suppose $\lambda \leq 2$. Then $\Lambda_{\lambda, q}$ is differentiable on $\mathbb{R}$, and $\Lambda_{\lambda, q}^{*}$ is strictly convex on $(0,1)$.

(c) Suppose $\lambda>2$, and let $Q$ be such that $\lambda=\lambda_{\mathrm{c}}(Q)$. Then $\Lambda_{\lambda, q}$ is differentiable except at the point $\nu$ satisfying $q e^{\nu}=Q$, at which point it has left (resp. right) derivative $\kappa^{-}(\lambda, Q)$ (resp. $\left.\kappa^{+}(\lambda, Q)\right)$. Also, $\Lambda_{\lambda, q}^{*}$ is strictly convex on $(0,1) \backslash\left(\kappa^{-}(\lambda, Q), \kappa^{+}(\lambda, Q)\right)$ and is linear on the deleted sub-interval. Furthermore, $\Lambda_{\lambda, q}^{*}$ is differentiable on $(0,1)$.

(d) Suppose that either $\lambda \leq 2$, or $\lambda>2$ and $\lambda \neq \lambda_{\mathrm{c}}(q)$. Then the derivative $\Lambda_{\lambda, q}^{\prime}(0)=\kappa(\lambda, q)$ exists, and $\Lambda_{\lambda, q}^{*}$ is strictly decreasing (resp. increasing) and strictly positive on $(0, \kappa(\lambda, q))$ (resp. $(\kappa(\lambda, q), 1))$, while $\Lambda_{\lambda, q}^{*}(\kappa(\lambda, q))=0$.

(e) Suppose $\lambda>2$ and $\lambda=\lambda_{\mathrm{c}}(q)$. Then $\Lambda_{\lambda, q}^{*}$ is strictly decreasing (resp. increasing) and strictly positive on $\left(0, \kappa^{-}(\lambda, q)\right)$ (resp. $\left.\left(\kappa^{+}(\lambda, q), 1\right)\right)$, while $\Lambda_{\lambda, q}^{*}(x)=$ 0 on $\left[\kappa^{-}(\lambda, q), \kappa^{+}(\lambda, q)\right]$. above,

We write $\mathcal{F}_{\lambda, q}$ for the set of 'exposed points' of $\Lambda_{\lambda, q}^{*}$, i.e., following (b) and (c)

$$
\mathcal{F}_{\lambda, q}= \begin{cases}(0,1) & \text { if } \lambda \leq 2 \\ (0,1) \backslash\left[\kappa^{-}(\lambda, Q), \kappa^{+}(\lambda, Q)\right] & \text { if } \lambda>2\end{cases}
$$

where $Q$ satisfies $\lambda=\lambda_{\mathrm{c}}(Q)$. The following LDP is a consequence of Thm 2.3.6 of Dembo and Zeitouni (1993). 
Theorem 8.3. Assume $\lambda, q>0$.

(a) For any closed subset $F$ of $\mathbb{R}$,

$$
\limsup _{n \rightarrow \infty}\left\{\frac{1}{n} \log \mathrm{P}\left(n^{-1} C_{n} \in F\right)\right\} \leq-\inf _{x \in F} \Lambda_{\lambda, q}^{*}(x) .
$$

(b) For any open subset $G$ of $\mathbb{R}$,

$$
\liminf _{n \rightarrow \infty}\left\{\frac{1}{n} \log \mathrm{P}\left(n^{-1} C_{n} \in G\right)\right\} \geq-\inf _{x \in G \cap \mathcal{F}_{\lambda, q}} \Lambda_{\lambda, q}^{*}(x) .
$$

Of especial interest are the cases when $F$ takes the form $[0, \alpha]$ or $[\beta, 1]$, analysed as follows using Theorem 8.3.

(a) Suppose $q \leq 2$. Then, as $n \rightarrow \infty$,

$$
\begin{aligned}
& \frac{1}{n} \log \mathrm{P}\left(C_{n} \leq \alpha n\right) \rightarrow-\Lambda_{\lambda, q}^{*}(\alpha), \\
& \frac{1}{n} \log \mathrm{P}\left(C_{n} \geq \beta n\right) \rightarrow-\Lambda_{\lambda, q}^{*}(\beta),
\end{aligned}
$$

whenever $0<\alpha \leq \kappa(\lambda, q) \leq \beta<1$.

(b) Suppose $q>2$ and $\lambda=\lambda_{\mathrm{c}}(q)$. Then (8.7) and (8.8) hold for $0<\alpha \leq \kappa^{-}(\lambda, q)<$ $\kappa^{+}(\lambda, q) \leq \beta<1$.

(c) Suppose $q>2$ and $\lambda \neq \lambda_{\mathrm{c}}(q)$. Let $Q$ be such that $\lambda=\lambda_{\mathrm{c}}(Q)$. We have that (8.7) and (8.8) hold for any $\alpha, \beta$ satisfying $0<\alpha \leq \kappa(\lambda, q) \leq \beta<1$ except possibly when

$$
\kappa^{-}(\lambda, Q)<\alpha \leq \kappa^{+}(\lambda, Q) \quad \text { or } \quad \kappa^{-}(\lambda, Q) \leq \beta<\kappa^{+}(\lambda, Q) .
$$

(Note that $\kappa^{+}(\lambda, Q)<\kappa(\lambda, q)$ if $Q<q$, and $\kappa^{-}(\lambda, Q)>\kappa(\lambda, q)$ if $Q>q$, so that only one of the cases in (8.9) can occur for any given value of $q$.) We do not know whether the appropriate inferior and superior limits of (8.7) and (8.8) coincide when (8.9) holds. Suppose that $Q<q$ and $\alpha$ satisfies the inequality of (8.9). In this case, we have from the LDP that (only)

$$
\begin{aligned}
-\Lambda_{\lambda, q}^{*}\left(\kappa^{-}(\lambda, Q)\right) & \leq \liminf _{n \rightarrow \infty}\left\{\frac{1}{n} \log \mathrm{P}\left(C_{n} \leq \alpha n\right)\right\} \\
& \leq \limsup _{n \rightarrow \infty}\left\{\frac{1}{n} \log \mathrm{P}\left(C_{n} \leq \alpha n\right)\right\} \leq-\Lambda_{\lambda, q}^{*}(\alpha) .
\end{aligned}
$$

Similar inequalities hold for $\mathrm{P}\left(C_{n} \geq \beta n\right)$ when $Q>q$ and $\beta$ satisfies the appropriate part of (8.9). A complete account of the limits in (8.7) and (8.8), under (8.9), would be provided by a more detailed analysis of first-order phase transitions than that presented in this paper.

We summarise the above facts before proving Theorem 8.2. Except in the special case when $\lambda=\lambda_{\mathrm{c}}(q)$ and $q>2$, the limit $\kappa=\lim _{n \rightarrow \infty}\left\{n^{-1} \mathrm{E}\left(C_{n}\right)\right\}$ exists, and $\mathrm{P}\left(C_{n} \leq \alpha n\right), \mathrm{P}\left(C_{n} \geq \beta n\right)$ decay at least exponentially when $\alpha<\kappa<\beta$. The exact (exponential) rate of decay can be determined except when the levels $\alpha n$ and 
$\beta n$ lie within the discontinuity of a first-order phase transition. In the exceptional case $\left(\lambda=\lambda_{c}(q)\right.$ and $\left.q>2\right)$, a similar conclusion holds when $\alpha<\kappa^{-}$and $\beta>\kappa^{+}$, for suitable $\kappa^{-}, \kappa^{+}$. (We do not know whether $\lim _{n \rightarrow \infty}\left\{n^{-1} \mathrm{E}\left(C_{n}\right)\right\}$ exists in the exceptional case.)

Since first-order transitions occur only when $q>2$, and since the critical $\lambda$-values of such $q$ fill the interval $(2, \infty)$, there is a weak sense in which the value $\lambda=2$ marks a singularity of the asymptotics of the random graph $G(n, \lambda / n, q)$. This holds for any value of $q$, including $q=1$. Therefore the usual Erdős-Rényi random graph senses the existence of a first-order phase transition in the random-cluster model, but only through its large deviations.

Proof of Theorem 8.2. (a) The function

$$
\zeta_{n}(\nu)=\frac{1}{n} \log Z\left(n, \lambda / n, e^{\nu}\right), \quad \nu \in \mathbb{R}
$$

is convex (as may be seen directly by differentiating twice and using Schwarz's inequality) and has derivative

$$
\zeta_{n}^{\prime}(\nu)=\frac{1}{n} \mathrm{E}\left(C_{n, e^{\nu}}\right)
$$

It follows that

$$
\zeta(\nu)=\lim _{n \rightarrow \infty} \zeta_{n}(\nu)=\phi\left(\lambda, e^{\nu}\right)
$$

is convex and satisfies

$$
\zeta_{n}^{\prime}(\nu) \rightarrow \zeta^{\prime}(\nu) \text { if } \zeta^{\prime}(\nu) \text { exists. }
$$

It may be verified from the formula for $\phi$ in Theorem 2.6 that $\zeta$ is differentiable on $(-\infty, \infty)$ except for pairs $(\lambda, \nu)$ such that $\lambda=\lambda_{\mathrm{c}}\left(e^{\nu}\right)$ and $e^{\nu}>2$, cf. the similar results for the $\lambda$-derivative $\partial \phi / \partial \lambda$ in Section 7. Moreover, with $q=e^{\nu}$, it is found that $\zeta^{\prime}=q \partial \phi / \partial q=\kappa(\lambda, q)$.

The explicit formulae for $\kappa(\lambda, q)$ may be derived in a number of ways. One is to calculate, using the value of $\phi$ in Theorem 2.6. Another is to use the colouring method, together with the fact that $\kappa(\lambda, q)$ is the limit, as $n \rightarrow \infty$, of the mean of $\left|C_{1}\right|^{-1}$, where $C_{1}$ is the order of the component of $G(n, \lambda / n, q)$ containing the vertex 1 (cf. Grimmett (1989), Thm 4.2). We note the implication that $\mathrm{E}\left(Z^{-1}\right)=1-\frac{1}{2} \lambda$, where $Z$ is the total size of a branching process with Poisson-distributed family-sizes having parameter $\lambda(<1)$. Cf. Jagers (1975), Thm 2.11.2.

The existence and values of the left and right derivatives $\kappa^{ \pm}(\lambda, q)$ follow from the convexity of $\zeta$.

(b,c) The differentiability of $\Lambda_{\lambda, q}$ follows from (8.1) and the above remarks. The strict convexity of $\Lambda_{\lambda, q}^{*}$ on $\mathcal{F}_{\lambda, q}$ (defined in (8.6)) follows from Lemma 2.3.9 of Dembo and Zeitouni (1993); the linearity of $\Lambda_{\lambda, q}^{*}$ on the deleted sub-interval (in (c)) follows by a computation involving (8.2). Using (8.3), we find that the derivative of $\Lambda_{\lambda, q}^{*}$ equals $\left(\Lambda_{\lambda, q}^{\prime}\right)^{-1}$, whenever the latter quantity is uniquely defined. It follows from (a) and the strict convexity of $\Lambda_{\lambda, q}$ that $\Lambda_{\lambda, q}^{*}$ is differentiable off the interval 
$\left[\kappa^{-}(\lambda, Q), \kappa^{+}(\lambda, Q)\right]$. Clearly it is differentiable in the interior of this interval, since it is linear there. Finally, by the above remarks, it has left and right derivatives equal to $\nu$ at both $x=\kappa^{-}, \kappa^{+}$.

(d) The existence and value of $\Lambda_{\lambda, q}^{\prime}(0)$ follow by (8.1) and part (a). The monotonicity of $\Lambda_{\lambda, q}^{*}$ will follow from the facts that

$$
\begin{array}{ll}
\Lambda_{\lambda, q}^{*}(x)=\sup _{\nu>0}\left\{\nu x-\Lambda_{\lambda, q}(\nu)\right\}, & x>\kappa(\lambda, q), \\
\Lambda_{\lambda, q}^{*}(x)=\sup _{\nu<0}\left\{\nu x-\Lambda_{\lambda, q}(\nu)\right\}, & x<\kappa(\lambda, q) .
\end{array}
$$

We check (8.10) and (8.11) next. Clearly $\Lambda_{\lambda, q}^{*}(\kappa(\lambda, q))=-\Lambda_{\lambda, q}(0)=0$, by (8.3). Now, if $x>\kappa(\lambda, q)$ and $\nu<0$, or if $x<\kappa(\lambda, q)$ and $\nu>0$, then

$$
\nu x-\Lambda_{\lambda, q}(\nu) \leq \nu \kappa(\lambda, q)-\Lambda_{\lambda, q}(\nu) \leq \Lambda_{\lambda, q}^{*}(\kappa(\lambda, q))=0,
$$

and (8.10) and (8.11) follow. The strict monotonicity is a consequence of strict convexity together with the fact that $\Lambda_{\lambda, q}^{*}(\kappa(\lambda, q))=0$.

(e) The proof is similar to that of $(d)$.

\section{The case $q<1$}

In our applications of the colouring method so far, we have assumed that $q>1$. Assume now that $q<1$, let $n_{1} \geq 1$, and colour each component of $G\left(n_{1}, p\right)=$ $G\left(n_{1}, p, 1\right)$ red with probability $r=q$, independently of the other components. By Lemma 3.1, the red graph is distributed as $G(N, p, q)$ on a random number $N$ of vertices. This observation will enable us to draw conclusions about $G(n, p, q)$ when $q<1$ (just as we did earlier when $q>1$ ) from the case $q=1$; this time we shall use the colouring in the 'opposite direction', going from 1 to $q$, rather than from $q$ to 1 . One major difference, when $q<1$, is that we must first show that $\mathrm{P}(N=n)$ may be made reasonably large by a suitable choice of $n_{1}$.

Given $p>0$, define $\gamma=\gamma\left(n_{1}\right)$ to be the largest root of $e^{-n_{1} p \gamma}=1-\gamma$, and note that $\gamma=0$ if and only if $n_{1} p \leq 1$. Next, given $n$ and $p=\lambda / n$, choose $n_{1} \geq n$ such that

$$
\left|\left\{\gamma\left(n_{1}\right)+\left(1-\gamma\left(n_{1}\right)\right) q\right\} n_{1}-n\right| \leq 1
$$

This is possible since changing $n_{1}$ to $n_{1}+1$ alters $\{\gamma+(1-\gamma) q\} n_{1}$ by at least $q$ and at most 2, as may easily be verified by calculating $d\left(\gamma n_{1}\right) / d n_{1}$ (temporarily regarding $n_{1}$ as a real variable).

In the sequel we fix $\lambda>0$, and let $c_{1}, c_{2}, \ldots$ denote suitable positive constants that may depend on $\lambda$ (and on $q$, which is fixed in this section). Note that $n_{1} p=$ $\lambda_{1}=\lambda /(\gamma+(1-\gamma) q)+\mathrm{O}\left(n^{-1}\right)$. It is easily verified that (for large $n$ at least) $\lambda_{1}>1$ (resp. $\lambda<1)$ if $\lambda>q$ (resp. $\lambda<q$ ). 
Lemma 9.1. Suppose that $p=\lambda / n$, where $\lambda \neq q$ is fixed, and let $n_{1}$ satisfy (9.1). Consider the standard random graph $G\left(n_{1}, p\right)=G\left(n_{1}, p, 1\right)$, colour each component red with probability $q$, independently of the other components, and let $N$ be the number of vertices in the red components. Then, for some positive constant $c_{1}=c_{1}(\lambda)$,

$$
\mathrm{P}(N=n) \geq c_{1} n^{-1 / 2}
$$

Proof. We consider the case $\lambda>q$, so that $\lambda_{1}>1$ for large $n$. The case $\lambda<q$ is similar but somewhat simpler, since it requires no special treatment for the largest component.

Let $T_{k}, k=1,2, \ldots, n_{1}$, denote the number of components of $G\left(n_{1}, p\right)$ that are trees of order $k$ (excluding the largest component, in the unlikely event that this is itself a tree), let $M=\sum_{2}^{n_{1}} k T_{k}$ be the number of vertices in the tree components having at least two vertices, let $L$ denote the order of the largest component, and let $U$ denote the number of vertices in the remaining components. Thus

$$
n_{1}=\sum_{k=1}^{n_{1}} k T_{k}+L+U=T_{1}+M+L+U \text {. }
$$

Moreover, let $T_{k}^{\prime}$ be the number of trees of order $k$ that are coloured red, let $M^{\prime}=$ $\sum_{2}^{n_{1}} k T_{k}^{\prime}$ be the number of vertices in the red tree-components, except isolated vertices, and let $U^{\prime}$ be the number of vertices in the red cyclic components except the giant. Hence

$$
N= \begin{cases}T_{1}^{\prime}+M^{\prime}+U^{\prime}+L & \text { if the giant is coloured red } \\ T_{1}^{\prime}+M^{\prime}+U^{\prime} & \text { otherwise. }\end{cases}
$$

We claim that (at least for large $n$ ) with probability at least $\frac{1}{2}$, all the following four inequalities hold:

$$
\begin{aligned}
T_{1} & \geq c_{2} n, \\
\sum_{k=2}^{n_{1}} k^{2} T_{k} & \leq c_{3} n, \\
\left|L-\gamma n_{1}\right| & \leq c_{4} n^{1 / 2}, \\
U & \leq c_{5} n^{1 / 2} .
\end{aligned}
$$

In fact, $T_{1}$, the number of isolated vertices in $G\left(n_{1}, p\right)$, is asymptotically normal with mean $\sim c_{6} n_{1} \sim c_{7} n$ and variance $\sim c_{8} n$; see Barbour (1982). Hence (9.4) holds with probability $1-\mathrm{o}(1)$ for any $c_{2}<c_{7}$. Similarly, $\sum_{k=2}^{n_{1}} k^{2} T_{k}$ is asymptotically normal with mean $\sim c_{9} n$ and variance $\sim c_{10} n$, by Pittel (1990), and (9.5) follows. Moreover, again by Pittel (1990), $\left(L-\gamma n_{1}\right) / n_{1}^{1 / 2}$ converges in distribution to a normal distribution, and thus (9.6) holds with probability at least $\frac{2}{3}$, say, if $c_{4}$ is sufficiently large. Finally, $U=\mathrm{O}_{p}(1)$, by Bollobás (1985), Thm VI.11, and thus (9.7) holds with probability $1-\mathrm{o}(1)$ for any $c_{5}>0$. 
Now condition on a fixed realization of the random graph $G\left(n_{1}, p\right)$ satisfying (9.4)-(9.7), and consider the colouring process. Note that, by (9.3), we have that $N=n$ if the giant component is coloured red and $T_{1}^{\prime}=n-M^{\prime}-U^{\prime}-L$; moreover, by (9.6) and (9.7),

$$
\begin{aligned}
\mid n-M^{\prime} & -U^{\prime}-L-q T_{1} \mid \\
& =\left|n-M^{\prime}-U^{\prime}-L-q\left(n_{1}-L-M-U\right)\right| \\
& \leq\left|n-\{\gamma+q-\gamma q\} n_{1}\right|+(1-q)\left|L-\gamma n_{1}\right|+\left|M^{\prime}-q M\right|+\left|U^{\prime}-q U\right| \\
& \leq 1+c_{4} \sqrt{n}+c_{5} \sqrt{n}+\left|M^{\prime}-q M\right| .
\end{aligned}
$$

By colouring the isolated vertices after all other components, we see, using independence and the fact that $T_{1}^{\prime}$ has the binomial distribution $\operatorname{Bin}\left(T_{1}, q\right)$ where $c_{2} n \leq T_{1} \leq n$ by $(9.4)$, that

$$
\begin{gathered}
\mathrm{P}(N=n) \geq \mathrm{P}(\text { the giant is red }) \mathrm{P}\left(\left|M^{\prime}-q M\right| \leq c_{11} n^{1 / 2}\right) \times \\
\quad \inf \left\{\mathrm{P}\left(T_{1}^{\prime}=j\right):\left|j-q T_{1}\right| \leq c_{12} n^{1 / 2}\right\} \\
\geq q \mathrm{P}\left(\left|M^{\prime}-q M\right| \leq c_{11} n^{1 / 2}\right) c_{13} n^{-1 / 2} .
\end{gathered}
$$

Moreover, the (conditional) expectation of $M^{\prime}$ equals $q M$, and the (conditional) variance is, using (9.5),

$$
\operatorname{Var}\left(M^{\prime}\right)=\sum_{k=2}^{n_{1}} k^{2} T_{k} q(1-q) \leq c_{3} n .
$$

Thus, by Chebyshev's inequality,

$$
\mathrm{P}\left(\left|M^{\prime}-q M\right| \leq c_{11} n^{1 / 2}\right) \geq 1-\frac{c_{3}}{c_{11}^{2}} \geq \frac{1}{2},
$$

for a suitable $c_{11}$.

Consequently, the probability that $N=n$, conditional on (9.4)-(9.7), is at least $c_{14} n^{-1 / 2}$. This implies that $\mathrm{P}(N=n) \geq \frac{1}{2} c_{14} n^{-1 / 2}$.

Note that Lemma 9.1 is false for the critical case $\lambda=q$. We conjecture that in this case $\mathrm{P}(N=n) \geq c n^{-2 / 3}$, which would enable us to extend also Theorem 2.3 to the case $q<1$.

Lemma 9.1 may be used as follows. Suppose that $\Pi$ is a graph property such that the probability that the red part of the standard random graph $G\left(n_{1}, p\right)$ has property $\Pi$ is sufficiently small, namely o $\left(n^{-1 / 2}\right)$. Then, by Lemmas 3.1 and 9.1 ,

$$
\begin{aligned}
& \mathrm{P}(G(n, p, q) \text { has property } \Pi) \\
& \quad=\mathrm{P}\left(\text { the red part of } G\left(n_{1}, p\right) \text { has property } \Pi \mid N=n\right) \\
& \leq \mathrm{P}\left(\text { the red part of } G\left(n_{1}, p\right) \text { has property } \Pi\right) / \mathrm{P}(N=n) \\
& \quad=\mathrm{o}(1) .
\end{aligned}
$$

This argument can be used to verify many claims about the structure of the graph $G(n, p, q)$, but we prefer to use the following sharper result. 
Lemma 9.2. Suppose that $\Pi$ is a graph property that is not affected by addition or deletion of isolated vertices. Let $n_{1}$ satisfy (9.1), and colour the components of $G\left(n_{1}, p\right)$ as above. Then

$$
\begin{aligned}
& \mathrm{P}(G(n, p, q) \text { has property } \Pi) \\
& \leq c_{15} n^{-1 / 2}+c_{16} \mathrm{P}\left(\text { the red part of } G\left(n_{1}, p\right) \text { has property } \Pi\right) .
\end{aligned}
$$

Proof. Let again $T_{1}$ be the number of isolated vertices and let $T_{1}^{\prime}$ be the number of these that are coloured red. We observe first that, by a straightforward application of Chebyshev's inequality, (9.4) holds with probability $1-\mathrm{O}\left(n^{-1}\right)$, and therefore

$$
\mathrm{P}\left(T_{1}^{\prime}<c_{17} n\right) \leq c_{18} n^{-1} .
$$

We do the colouring of $G\left(n_{1}, p\right)$ in two stages. First colour all components with at least two vertices and let $G^{\prime}$ denote the resulting coloured graph; then colour also the isolated vertices. Let $G_{r}$ be the red part of the final graph and $G_{r}^{\prime}$ the red part of $G^{\prime}$. By assumption, $G_{r}$ has property $\Pi$ if and only if $G_{r}^{\prime}$ has. Now, condition on $G^{\prime}$ and the colours of components thereof; note that this fixes $G_{r}^{\prime}$ and $T_{1}$. If $G^{\prime}$ is such that $T_{1} \geq c_{1} n$, and $m$ is the number of red vertices in $G^{\prime}$, then

$$
\mathrm{P}\left(N=n \mid G^{\prime}\right)=\mathrm{P}\left(T_{1}^{\prime}=n-m \mid G^{\prime}\right) \leq c_{19} T_{1}^{-1 / 2} \leq c_{20} n^{-1 / 2},
$$

because $T_{1}^{\prime}$ has the binomial distribution $\operatorname{Bin}\left(T_{1}, q\right)$. Consequently,

$$
\begin{aligned}
\mathrm{P}\left(G_{r}\right. & \text { has property } \Pi, N=n) \\
& \leq \mathrm{P}\left(T_{1}<c_{1} n\right)+\mathrm{P}\left(G_{r}^{\prime} \text { has property } \Pi, T_{1} \geq c_{1} n, N=n\right) \\
& \leq \mathrm{P}\left(T_{1}<c_{1} n\right)+c_{20} n^{-1 / 2} \mathrm{P}\left(G_{r}^{\prime} \text { has property } \Pi, T_{1} \geq c_{1} n\right) \\
& \leq c_{18} n^{-1}+c_{20} n^{-1 / 2} \mathrm{P}\left(G_{r} \text { has property } \Pi\right) .
\end{aligned}
$$

The estimate (9.10) now follows by Lemma 9.1 and the fact that

$$
\begin{aligned}
\mathrm{P}(G(n, p, q) \text { has property } \Pi) & =\mathrm{P}\left(G_{r} \text { has property } \Pi \mid N=n\right) \\
& =\frac{\mathrm{P}\left(G_{r} \text { has property } \Pi, N=n\right)}{\mathrm{P}(N=n)} .
\end{aligned}
$$

The claims in Theorems 2.1 and 2.2 for $q<1$ now follow almost immediately from the corresponding results for $G\left(n_{1}, p, 1\right)$. In obtaining these theorems, we fix $n$ and $\lambda$, we set $p=\lambda / n$ and choose $n_{1}$ to satisfy (9.1), and we then let $n \rightarrow \infty$. For the supercritical case we also need the easy fact that

$$
\mathrm{P}(N=n \text {, and the giant component is not coloured red })=\mathrm{o}\left(n^{-1 / 2}\right),
$$

when $n_{1}$ is chosen to satisfy (9.1). For the assertion on the largest tree, we use the fact that for every fixed $m$, a.e. $G\left(n_{1}, p, 1\right)$ contains $m$ trees of the given size, see Bollobás (1985), Thms V.10 and VI.11. Thus the probability that the red part contains no such tree is at most $(1-q)^{m}+\mathrm{o}(1)$, which can be made arbitrarily small.

The same method implies the conclusion of Theorem 5.1 when $q<1$ and $\lambda \neq q$. The calculations in Section 6 hold without change, which implies the asymptotic formula for the partition function in Theorem 2.6 when $\lambda \neq q<1$. By certain properties of monotonicity of the partition function as a function of either $q$ or $\lambda$, we have that Theorem 2.6 holds also for $\lambda=q$. 


\section{External field}

In the classical paradigm of phase transition, a non-zero external field $h$ is added, and the order parameter is defined by

$$
M(\beta)=\lim _{h \downarrow 0} \frac{d \phi}{d h}
$$

where $\phi$ is the free energy and $\beta$ is the reciprocal of temperature; see Thompson (1972). An 'external field' may be added to the random-cluster model in the following way, reminiscent of an associated Potts model. Let $0<p, h<1, q>0$, and define the random graph $G(n, p, q, h)$ as follows. There are $n+1$ vertices labelled $0,1,2, \ldots, n$. Any subset $E$ of edges of the corresponding complete graph $K_{n+1}$ has weight

$$
\widetilde{P}(E ; n, p, q, h)=h^{\left|E^{\prime}\right|}(1-h)^{n-\left|E^{\prime}\right|} p^{\left|E^{\prime \prime}\right|}(1-p)^{\left(\begin{array}{c}
n \\
2
\end{array}\right)-\left|E^{\prime \prime}\right|} q^{c(E)},
$$

where $E^{\prime}$ is the subset of $E$ containing edges incident with 0 , and $E^{\prime \prime}=E \backslash E^{\prime}$; cf. (1.1). This weighting function gives rise to a probability measure

$$
\mathrm{P}(E ; n, p, q, h)=\frac{\widetilde{P}(E ; n, p, q, h)}{Z(n, p, q, h)}
$$

where

$$
Z(n, p, q, h)=\sum_{E} \widetilde{P}(E ; n, p, q, h)
$$

as before. The colouring method of Section 3 may be applied in order to analyse this model. We do not describe this in detail, but summarise some conclusions as follows. For simplicity we consider only the case $q \geq 1$.

Theorem 10.1. Assume $q \geq 1$ and let $\lambda>0$. The free energy

$$
\phi(\lambda, q, h)=\lim _{n \rightarrow \infty}\left\{\frac{1}{n} \log Z(n, \lambda / n, q, h)\right\}
$$

exists for $h>0$, and is given by

$$
\phi(\lambda, q, h)=\frac{g(\theta(\lambda, h))}{2 q}-\frac{q-1}{2 q} \lambda+\log q+\frac{(q-1)(2-\theta(\lambda, h))}{2 q} \log (1-h)
$$

where $g$ is defined in (6.17) and

$$
(1-h) e^{-\lambda \theta(\lambda, h)}=\frac{1-\theta(\lambda, h)}{1+(q-1) \theta(\lambda, h)}
$$

if there are several positive roots to (10.3) we choose the one that maximizes (10.2). Moreover, when this defines $\theta(\lambda, h)$ uniquely, if $\Theta_{n} n$ is the number of vertices in the largest component, then $\Theta_{n} \stackrel{\mathrm{P}}{\rightarrow} \theta(\lambda, h)$. Similarly, if $\Psi_{n} n$ is the number of 
edges in $G(n, p, q, h)$ that are not adjacent to 0 , and $\Xi_{n} n$ is the number of these that belong to the largest component, then (5.5) and (5.6) hold; the number of edges adjacent to 0 (which for a.e. $G(n, p, q, h)$ belongs to the largest component) is $n h\left\{\Theta_{n}+q^{-1}\left(1-\Theta_{n}\right)\right\}+\mathrm{o}_{\mathrm{p}}(n)$.

Sketch of proof. For $q=1$, we obtain $G(n, p, 1, h)$ from the ordinary random graph $G(n, p)$ by adding an extra vertex 0 and adding edges between 0 and the other vertices with probability $h$, all edges being added independently. The result follows easily from standard results for $G(n, p)$.

For $q>1$ we argue as in Sections 3-6 with only minor differences. In (6.1) et seq., $c(j, k, l)$ is replaced by $c(j, k, l, s)$, the number of choices for the cyclic component having $s$ edges incident with the new vertex 0 , and $m$ and $l$ are counts of edges which are not incident with 0 . There is also the term $h^{s}(1-h)^{n-s}$.

Note that $\theta(\lambda, h)>0$ for $h>0$, so we do not have to consider the case $\theta=0$.

It follows from (10.2), (10.3), and (6.17) by an elementary calculation that

$$
\frac{\partial}{\partial h} \phi(\lambda, q, h)=-\frac{1}{1-h} \frac{q-1}{q}(1-\theta(\lambda, q)) .
$$

This confirms the physical paradigm, in which the zero-field magnetisation is obtained as the derivative of the free energy with respect to the external field $h$, evaluated at $h=0$ (subject to changes of scale and location). A similar argument may be followed if $q<1$.

\section{Fixed edge-number model}

Finally we consider a version of the random-cluster model having a fixed number of edges. Assume that $q>0$ and that $m$ and $n$ are integers with $0 \leq m \leq\left(\begin{array}{l}n \\ 2\end{array}\right)$. For any subset $E$ of the edge-set of $K_{n}$, we set

$$
\widetilde{P}(E ; n, m, q)=\left(\begin{array}{c}
n \\
2 \\
m
\end{array}\right){ }^{-1} q^{c(E)} .
$$

As before, we let

$$
\mathrm{P}(E ; n, m, q)=\frac{\widetilde{P}(E ; n, m, q)}{Z(n, m, q)}, \quad Z(n, m, q)=\sum_{E} \widetilde{P}(E ; n, m, q) .
$$

The function $\mathrm{P}(\cdot ; n, m, q)$ is a probability measure, which agrees when $q=1$ with the measure describing a 'fixed edge-number' random graph $G(n, m)$ on $n$ vertices and $m$ edges; see Bollobás (1985). Note that $\widetilde{P}$ has been defined in (11.1) in such a way that $Z(n, m, 1)=1$.

The graph $G(n, m, q)$ describes the conditional distribution of $G(n, p, q)$, given that the number of edges of the latter graph is $m$. We might therefore expect that $G(n, m, q)$ and $G(n, p, q)$ have broadly similar behaviour, so long as the parameters 
$m, p, q$ are chosen in such a way that $p=\lambda / n$ and $m \sim \psi(\lambda) n$ where $\psi(\lambda)=$ $\lambda\left\{1+(q-1) \theta(\lambda)^{2}\right\} /(2 q)$; see Theorem 5.2. This turns out indeed to be the case, but with one important extra point to note. When $q>2$, the function $\psi(\lambda)$ has a jump discontinuity at $\lambda=\lambda_{\mathrm{c}}(q)$, and therefore $\psi$ does not take all positive values. Hence there are values of $m$, including $m=\left\lfloor\frac{1}{2} n\right\rfloor$ (see part (c) in Section 7), for which $G(n, m, q)$ has no counterpart amongst $G(n, p, q)$ when $q>2$. We point out that some of our results, in the case $q>2$, depend on the validity of the following Hypothesis 11.5. Numerical work (using Mathematica) supports this hypothesis, and we can prove it when either $q<2+\epsilon$ or $q>\epsilon^{-1}$ (for some small positive $\epsilon$ ), but we have no general proof.

In this section we summarise the structure of $G(n, m, q)$ in the limit as $m / n \rightarrow \mu$ where $\mu \in(0, \infty)$. For simplicity we consider only $q \geq 1$.

Theorem 11.1. Let $q \geq 1$ and assume that $m / n \rightarrow \mu(\in(0, \infty))$ as $n \rightarrow \infty$.

(a) Assume $q \leq 2$. Then $G(n, m, q)$ has largest component of order $\theta(\mu, q) n+$ $\mathrm{o}_{\mathrm{p}}(n)$, where

$$
\theta(\mu, q) \begin{cases}=0 & \text { if } \mu \leq \frac{1}{2} \\ >0 & \text { if } \mu>\frac{1}{2}\end{cases}
$$

and $\theta\left(\frac{1}{2}+, q\right)=0$.

(b) Assume that $q>2$, and that Hypothesis 11.5 is valid. There exists $\mu_{\mathrm{c}}=\mu_{\mathrm{c}}(q)$ such that $G(n, m, q)$ has largest component of order $\theta(\mu, q) n+\mathrm{o}_{\mathrm{p}}(n)$, where

$$
\theta(\mu, q) \begin{cases}=0 & \text { if } \mu<\mu_{\mathrm{c}}(q) \\ >0 & \text { if } \mu>\mu_{\mathrm{c}}(q) .\end{cases}
$$

Furthermore $\theta\left(\mu_{\mathrm{c}}(q)+, q\right)>0$.

We sketch a proof of this in the remainder of this section; further details of the structure of $G(n, m, q)$ may be found similarly. Within this proof, we shall see how $\mu_{\mathrm{c}}(q)$ and $\theta\left(\mu_{\mathrm{c}}(q)+, q\right)$ may be calculated when $q>2$, but we have been unable to obtain analogues of the closed formulae given earlier for $G(n, p, q)$. Further information about $\mu_{\mathrm{c}}(q)$ and $\theta(\mu, q)$ is provided after Hypothesis 11.5. This hypothesis is used in part of the proof of Theorem 11.1(b). It is unsatisfactory that we have no proof of the hypothesis, although numerical experiments using Mathematica encourage us to believe it to be valid.

Proof. We shall make use of the same colouring argument as before; with a slight modification of the proof of Lemma 3.1, we find that the red subgraph of $G(n, m, q)$, conditional on having $N$ vertices and $M$ edges, is distributed as $G(N, M, r q)$. Again we take $q>1$, and let $r=q^{-1}$. Lemma 3.2 holds without changes in the new setting. Therefore, with $\Theta_{n}$ and $\Xi_{n}$ as before, and with $m \sim \mu n$, the red part has either $\left\{\Theta_{n}+r\left(1-\Theta_{n}\right)\right\} n+\mathrm{o}_{\mathrm{p}}(n)$ vertices and $\left\{\Xi_{n}+r\left(\mu-\Xi_{n}\right)\right\} n+\mathrm{o}_{\mathrm{p}}(n)$ edges, including a component with $\Theta_{n} n$ vertices and $\Xi_{n} n$ edges, or $r\left(1-\Theta_{n}\right) n+o_{\mathrm{p}}(n)$ 
vertices and $r\left(\mu-\Xi_{n}\right) n+\mathrm{o}_{\mathrm{p}}(n)$ edges and no large component. We set

$$
\begin{aligned}
& \Theta_{n}^{\prime}=\frac{\Theta_{n}}{\Theta_{n}+r\left(1-\Theta_{n}\right)}=\frac{q \Theta_{n}}{1+(q-1) \Theta_{n}}, \\
& \Psi_{n}^{\prime}=\frac{\Xi_{n}+r\left(\mu-\Xi_{n}\right)}{\Theta_{n}+r\left(1-\Theta_{n}\right)}=\frac{\mu+(q-1) \Xi_{n}}{1+(q-1) \Theta_{n}}, \\
& \Xi_{n}^{\prime}=\frac{\Xi_{n}}{\Theta_{n}+r\left(1-\Theta_{n}\right)}=\frac{q \Xi_{n}}{1+(q-1) \Theta_{n}},
\end{aligned}
$$

and we write $X_{n} \stackrel{\mathrm{P}}{\approx} Y_{n}$ if $X_{n}-Y_{n} \stackrel{\mathrm{P}}{\rightarrow} 0$ as $n \rightarrow \infty$. From the first case above, it follows that

$$
1-\Theta_{n}^{\prime} \stackrel{\mathrm{P}}{\approx} e^{-\Lambda_{n}^{\prime} \Theta_{n}^{\prime}}
$$

where $\Lambda_{n}^{\prime}=2 \Psi_{n}^{\prime}$ and we have used the first statement in the proof of Lemma 4.2, in a version for $G(n, m, 1)$; see Bollobás (1985), Thm VI.11. Applying the analogue of (5.6) for $G(n, m, 1)$ to the second case above, we obtain that

$$
\Xi_{n}^{\prime} \stackrel{\mathrm{P}}{\approx} \Lambda_{n}^{\prime} \Theta_{n}^{\prime}\left(1-\frac{1}{2} \Theta_{n}^{\prime}\right)
$$

Therefore

$$
\begin{aligned}
\mu & =\left\{1+(q-1) \Theta_{n}\right\} \Psi_{n}^{\prime}-(q-1) \Xi_{n} \\
& \stackrel{\mathrm{P}}{\approx}\left\{1+(q-1) \Theta_{n}\right\} \Psi_{n}^{\prime}-\frac{q-1}{q}\left\{1+(q-1) \Theta_{n}\right\}\left(2-\Theta_{n}^{\prime}\right) \Psi_{n}^{\prime} \Theta_{n}^{\prime} \\
& =\left\{1+(q-1) \Theta_{n}\right\} \Psi_{n}^{\prime}-\frac{q-1}{q}\left\{2+2(q-1) \Theta_{n}-q \Theta_{n}\right\} \Psi_{n}^{\prime} \Theta_{n}^{\prime}
\end{aligned}
$$

by $(11.5),(11.6),(11.8)$, and (11.4). It follows that

$$
\begin{aligned}
q \Theta_{n} \mu & \stackrel{\mathrm{P}}{\approx}\left\{1+(q-1) \Theta_{n}\right\}^{2} \Theta_{n}^{\prime} \Psi_{n}^{\prime}-(q-1)\left\{2 \Theta_{n}+(q-2) \Theta_{n}^{2}\right\} \Theta_{n}^{\prime} \Psi_{n}^{\prime} \\
& =\left\{1+(q-1) \Theta_{n}^{2}\right\} \Theta_{n}^{\prime} \Psi_{n}^{\prime} \\
& \stackrel{\mathrm{P}}{\approx}-\frac{1}{2}\left\{1+(q-1) \Theta_{n}^{2}\right\} \log \left(1-\Theta_{n}^{\prime}\right) \\
& =\frac{1}{2}\left\{1+(q-1) \Theta_{n}^{2}\right\} \log \left(\frac{1+(q-1) \Theta_{n}}{1-\Theta_{n}}\right)
\end{aligned}
$$

by (11.4) and (11.7). Thus we arrive at the analogue of Lemma 4.2 and Theorem 5.1 .

Lemma 11.2. Suppose $q \geq 1$ and $m / n \rightarrow \mu$ as $n \rightarrow \infty$. Then, as $n \rightarrow \infty$,

$$
\begin{aligned}
& \mu \Theta_{n}-\frac{1}{2 q}\left\{1+(q-1) \Theta_{n}^{2}\right\} \log \left(\frac{1+(q-1) \Theta_{n}}{1-\Theta_{n}}\right) \stackrel{\mathrm{P}}{\rightarrow} 0, \\
& \Xi_{n}-\frac{1}{q}\left\{\left(1+\left(\frac{1}{2} q-1\right) \Theta_{n}\right\} \log \left(\frac{1+(q-1) \Theta_{n}}{1-\Theta_{n}}\right) \stackrel{\mathrm{P}}{\rightarrow} 0 .\right.
\end{aligned}
$$


Equation (11.10) follows from (11.9), and (11.11) follows similarly by (11.4)(11.8). By (11.10), for $\epsilon>0$, a.e. $G(n, m, q)$ has $\Theta_{n}$ within distance $\epsilon$ of the set of roots of the equation

$$
\theta \mu=\frac{1+(q-1) \theta^{2}}{2 q} \log \left(\frac{1+(q-1) \theta}{1-\theta}\right) .
$$

If $\Theta_{n}$ is close to the root $\theta$, then by (11.11) $\Xi_{n}$ is close to

$$
\xi(\lambda, q)=\frac{\lambda \theta}{q}\left\{1+\left(\frac{1}{2} q-1\right) \theta\right\}
$$

where $\lambda=\lambda(\theta)$ is defined for $\theta \neq 0$ by

$$
\lambda=\frac{1}{\theta} \log \left(\frac{1+(q-1) \theta}{1-\theta}\right) ;
$$

cf. (2.3) and (5.6). In this sense, $G(n, m, q)$ and $G(n, \lambda / n, q)$ have comparable properties when $\Theta_{n}$ is close to a positive root $\theta$ of (11.12). The total numbers of edges in these random graphs match each other also, since the above equation for $\lambda$ may be written in the form

$$
\mu=\frac{\lambda}{2 q}\left\{1+(q-1) \theta^{2}\right\}
$$

cf. (5.5).

In order to ascertain which root of (11.12) is the correct limit for $\Theta_{n}$, we prove an analogue of Lemma 4.1.

Lemma 11.3. If $q \geq 1$ and $m / n \rightarrow \mu$ where $\mu>\frac{1}{2}$, then there exists $\theta_{0}(>0)$ such that $\Theta_{n} \geq \theta_{0}$ for a.e. $G(n, m, q)$.

Proof. If no such $\theta_{0}$ exists then, for every $\epsilon>0$, there exists $\delta(>0)$ such that $\mathrm{P}\left(\Theta_{n} \leq \epsilon\right)>\delta$ for all large $n$. We have from (11.11) that, for every $\eta>0$, there exists $\epsilon(>0)$, such that $\mathrm{P}\left(\Theta_{n} \leq \epsilon, \Xi_{n}>\eta\right) \rightarrow 0$ as $n \rightarrow \infty$. With $\delta$ chosen accordingly, it follows that

$$
\mathrm{P}\left(\Xi_{n} \leq \eta, \Theta_{n} \leq \epsilon\right)>\frac{1}{2} \delta
$$

for all large $n$. For such $n$, and with probability at least $\frac{1}{3}(1-r) \delta$, our usual colouring method results in a red subgraph with $N$ vertices and $M$ edges and no component larger than $n^{3 / 4}$, where

$$
\begin{aligned}
& N=r\left(n-\Theta_{n} n\right)+\mathrm{o}(n) \in r n(1-\epsilon, 1)+\mathrm{o}(n), \\
& M=r\left(m-\Xi_{n} n\right)+\mathrm{o}(n) \geq r(\mu-\eta) n+\mathrm{o}(n),
\end{aligned}
$$

and therefore $n^{3 / 4}=\mathrm{O}\left(N^{3 / 4}\right)$. Now $M / N \geq \mu-2 \eta$ for all large $n$. Suppose $\mu>\frac{1}{2}$ and pick $\eta(>0)$ such that $\mu-2 \eta>\frac{1}{2}$. The non-existence of a large component in the red subgraph contradicts the existence of a giant component in a.e. 
$G\left(n^{\prime},(\mu-2 \eta) n^{\prime}, 1\right)$.

In the light of Lemma 11.3, we shall be interested only in values of $\mu$ and roots $\theta$ of (11.12) satisfying

$$
0 \leq \theta<1 \text {, and either } \theta>0 \text {, or } \theta=0 \text { and } \mu \leq \frac{1}{2} \text {. }
$$

Let $\mu(>0)$ and $q(\geq 1)$ be fixed, and let $\theta$ be a root of (11.12) satisfying (11.14). We define

$$
\lambda=\frac{2 q \mu}{1+(q-1) \theta^{2}},
$$

as in (11.13), so that $\lambda$ is a root of (2.3); it is the case that $\lambda \leq q$ if $\theta=0$. We define $\xi$ and $\psi$ as in (6.3) and (6.4) by

$$
\begin{aligned}
& \xi=\frac{\lambda \theta}{q}\left\{1+\left(\frac{1}{2} q-1\right) \theta\right\}=\frac{1}{q}\left\{1+\left(\frac{1}{2} q-1\right) \theta\right\} \log \left(\frac{1+(q-1) \theta}{1-\theta}\right), \\
& \psi=\xi+\frac{\lambda}{2 q}(1-\theta)^{2}=\mu .
\end{aligned}
$$

As in Section 6 , we write $\widetilde{P}(j, k, l ; n, m, q)$ for the sum of all $\widetilde{P}(E ; n, m, q)$ over all edge-sets $E$ having a cyclic part with $j$ components, $k$ vertices, and $l$ edges. We assume that $m / n \rightarrow \mu, j / n \rightarrow 0, k / n \rightarrow \theta$, and $l / n \rightarrow \psi$. The present $\widetilde{P}$ differs from the $\widetilde{P}$ in $(6.1)$ by a factor

$$
\begin{aligned}
\left(\begin{array}{c}
\left(\begin{array}{c}
n \\
2
\end{array}\right) \\
m
\end{array}\right)^{-1}\left(\frac{\lambda}{n}\right)^{-m}\left(1-\frac{\lambda}{n}\right)^{m-\left(\begin{array}{c}
n \\
2
\end{array}\right)} & =\left(\frac{n^{2}}{2} \frac{e}{m} \frac{\lambda}{n}\right)^{-m} \exp \left(\frac{1}{2} \lambda n+\mathrm{o}(n)\right) \\
& =\left(\frac{2 \mu}{e \lambda}\right)^{m} \exp \left(\frac{1}{2} \lambda n+\mathrm{o}(n)\right) \\
& =\left(\frac{1+(q-1) \theta^{2}}{e q}\right)^{m} \exp \left(\frac{1}{2} \lambda n+\mathrm{o}(n)\right)
\end{aligned}
$$

by (11.15). In combination with (6.16), this yields

$$
\begin{aligned}
& \widetilde{P}(j, k, l ; n, m, q) \leq \exp \left\{n \left[\frac{g(\theta)}{2 q}-\frac{q-1}{2 q} \lambda+\log q+\mu \log \left\{1+(q-1) \theta^{2}\right\}\right.\right. \\
&\left.\left.-\mu-\mu \log q+\frac{1}{2} \lambda+o(1)\right]\right\} \\
&=\exp \left\{n\left[\frac{J(\theta)}{2 q}+(1-\mu) \log q+o(1)\right]\right\}
\end{aligned}
$$


where

$$
\begin{aligned}
J(\theta)= & g(\theta)+2 q \mu \log \left\{1+(q-1) \theta^{2}\right\}-2 q \mu+\lambda \\
= & g(\theta)+2 q \mu \log \left\{1+(q-1) \theta^{2}\right\}-\lambda(q-1) \theta^{2} \\
= & \frac{1+(q-1) \theta^{2}}{\theta} \log \left(\frac{1+(q-1) \theta}{1-\theta}\right) \log \left\{1+(q-1) \theta^{2}\right\} \\
& \quad-2(q-1)(1-\theta) \log (1-\theta)-2\{1+(q-1) \theta\} \log \{1+(q-1) \theta\}
\end{aligned}
$$

by (11.15) and (2.3). Moreover there is at least one choice of the sequences $j, k, l$ for which equality holds in (11.18).

Lemma 6.1 and its proof are valid in the present setting as before, and the remainder of Section 6 may be followed to deduce that $\Theta_{n} \stackrel{\mathrm{P}}{\rightarrow} \theta^{*}$ where $\theta^{*}$ is the root of (11.12) satisfying (11.14) for which $J(\theta)$ is a maximum.

This identifies the order of the largest component of a.e. $G(n, m, q)$ when $m / n \rightarrow$ $\mu$. The position and type of the phase transition are then specified in terms of the following properties of the functions $J$ and

$$
\mu(\theta)=\frac{1+(q-1) \theta^{2}}{2 q \theta} \log \left(\frac{1+(q-1) \theta}{1-\theta}\right), \quad 0<\theta<1,
$$

where $\mu(0)=\frac{1}{2}$; cf. (11.12).

Lemma 11.4. The function $\mu$ satisfies the conclusions of Lemma 2.4 except inasmuch as $\mu(0+)=\frac{1}{2}$.

Proof. We have that

$$
\mu(\theta)=\left\{\frac{1}{2 q \theta} \log \left(\frac{1+(q-1) \theta}{1-\theta}\right)\right\}+\left\{\frac{q-1}{2 q} \theta \log \left(\frac{1+(q-1) \theta}{1-\theta}\right)\right\}
$$

is the sum of two convex functions, and is therefore convex on $(0,1)$; the first function in (11.21) is convex as in Lemma 2.4, and an easy computation shows that the second function has a second derivative which is strictly positive on $(0,1)$. It is easy to check that $\mu^{\prime}(0)=\frac{1}{4}(2-q)$ and $\mu^{\prime \prime}(0)>0$, and the remaining claims follow.

This lemma has the following consequences. If $1 \leq q \leq 2$ and $\mu>0$, there is a unique solution $\theta=\theta(\mu, q)$ to (11.12) subject to (11.14), and $\theta(\mu, q)=0$ if and only if $\mu \leq \frac{1}{2}$. Furthermore $\theta\left(\frac{1}{2}+, q\right)=0$, and the first part of Theorem 11.1 follows with $\theta(\mu, q)$ defined accordingly.

Assume now that $q>2$. There exists $\mu_{\min }$ satisfying $0<\mu_{\min }<\frac{1}{2}$ such that (11.12) has the single root $\theta=0$ when $\mu<\mu_{\min }$, two roots $\left(0\right.$ and $\left.\theta_{\text {max }}\right)$ when $\mu=\mu_{\min }$, three roots $\left(0, \theta_{1}\right.$, and $\left.\theta_{\max }\right)$ when $\mu_{\min }<\mu<\frac{1}{2}$, and a unique positive root $\theta_{\max }$ when $\mu \geq \frac{1}{2}$. It follows in particular that part (b) of Theorem 11.1 holds when $\mu<\mu_{\min }$ or $\mu>\frac{1}{2}$, and with $\theta(\mu, q)$ defined respectively as 0 and $\theta_{\max }$. This completes the analogue of Theorem 4.3.

We turn now to the case $q>2$ and $\mu_{\text {min }} \leq \mu \leq \frac{1}{2}$. 


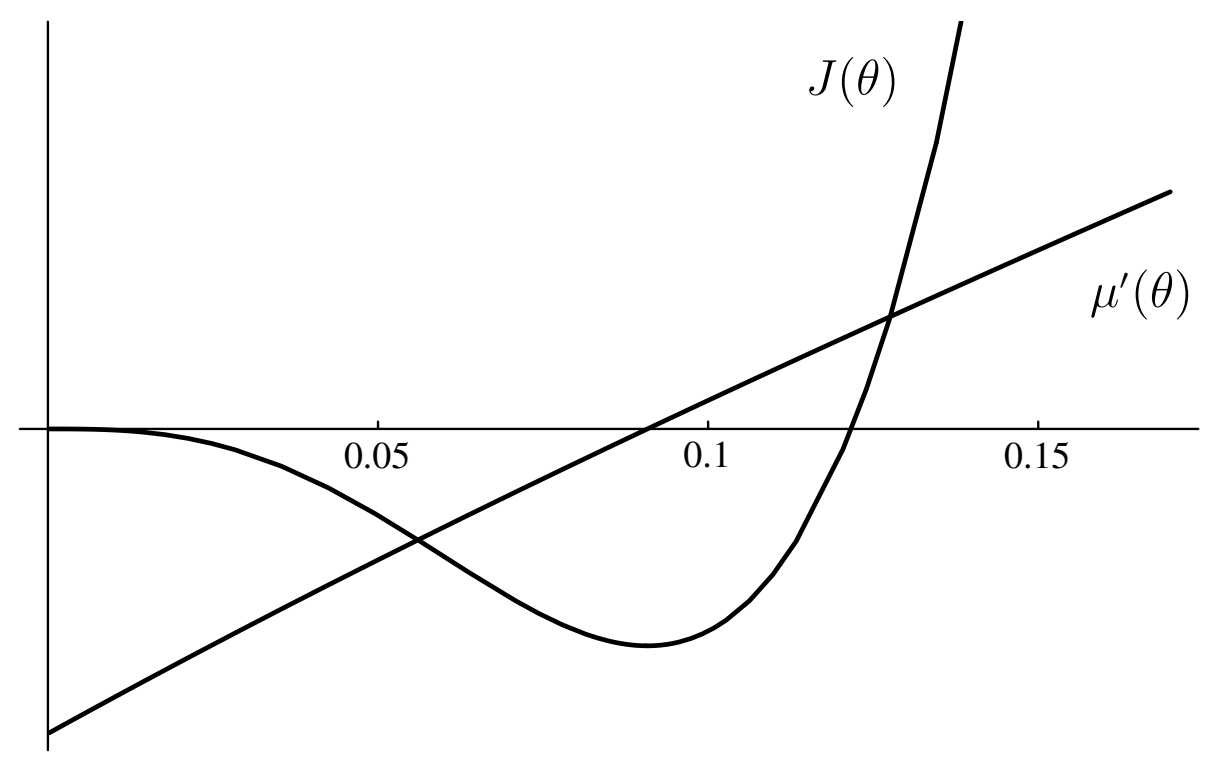

Figure 2. A plot of the function $J(\theta)$ and the derivative $\mu^{\prime}(\theta)$, when $q=3$. Note that the zero of $\mu^{\prime}$ (i.e., the minimum of $\mu$ ) lies to the left of the unique zero of $J$ in $(0,1)$. We believe that the general features of this figure persist for any $q$ satisfying $q>2$.

Hypothesis 11.5. Assume $q>2$. The function $J(\theta)$ has a unique zero on $(0,1)$, at a point $\theta_{0}$. We have that $J$ is negative on $\left(0, \theta_{0}\right)$, and positive on $\left(\theta_{0}, 1\right)$. Furthermore $\theta_{0}>\theta_{\min }$ where $\theta_{\min }$ is the value of $\theta$ at which $\mu(\theta)$ is a minimum.

With the aid of this hypothesis, and the continuity of $J$ on $[0,1)$, we obtain that $\theta(\mu, q)$ in Theorem 11.1(b) is given by

$$
\theta(\mu, q)= \begin{cases}0 & \text { if } \mu<\mu_{\mathrm{c}}(q) \\ \theta_{\max }(\mu) & \text { if } \mu>\mu_{\mathrm{c}}(q)\end{cases}
$$

where $\mu_{\mathrm{c}}(q)=\mu\left(\theta_{0}\right)$. Furthermore $\theta\left(\mu_{\mathrm{c}}(q)+, q\right)=\theta_{0}>0$, which indicates the first-order phase transition.

As an encouragement to the reader to believe in Hypothesis 11.5, we present in Figure 2 a plot of $J$ and the derivative $\mu^{\prime}$ when $q=3$. We have verified the general features of this picture, using Mathematica, for a variety of values of $q$. Finally, we have a proof of the hypothesis for certain values of $q$, namely when either $q<2+\epsilon$ or $q>\epsilon^{-1}$ for some small positive $\epsilon$. (It is the case that $\theta_{\min } \sim q^{-1 / 2}$ and $\theta_{0} \sim \gamma q^{-1 / 2}$ as $q \rightarrow \infty$, where $\gamma>1$ is the root of $\left(1+\gamma^{2}\right) \log \left(1+\gamma^{2}\right)=2 \gamma^{2}$.)

\section{Acknowledgements}

This work was aided by the EU under contract CHRX-CT93-0411. G.R.G. acknowledges partial support from the Isaac Newton Institute, University of Cambridge, and by the SERC under grant GR G59981. S.J. acknowledges support by the Göran Gustafsson Foundation for Research in Natural Sciences and Medicine. 


\section{REFERENCES}

Barbour, A. D. (1982), Poisson convergence and random graphs, Mathematical Proceedings of the Cambridge Philosophical Society 92, 349-359.

Bollobás, B. (1984), The evolution of random graphs, Transactions of the American Mathematical Society 286, 257-274.

Bollobás, B. (1985), Random Graphs, Academic Press, London.

Britikov, V. E. (1988), The asymptotics for the number of forests of non-rooted trees., Matem. Zametki 43, 672-684 (Russian); English transl., Mathematical Notes 43, 387-394.

Dembo, A. and Zeitouni, O. (1993), Large Deviation Techniques, Jones and Bartlett, Boston.

Edwards, R. G. and Sokal, A. D. (1988), Generalization of the Fortuin-Kasteleyn-SwendsenWang representation and Monte Carlo algorithm, The Physical Review D 38, 2009-2012.

Grimmett, G. R. (1989), Percolation, Springer-Verlag, Berlin.

Grimmett, G. R. (1994a), The random-cluster model, Probability, Statistics and Optimisation (F. P. Kelly, ed.), John Wiley \& Sons, Chichester, pp. 49-63.

Grimmett, G. R. (1994b), Percolative problems, Probability and Phase Transition (G. R. Grimmett, ed.), Kluwer, Dordrecht, pp. 69-86.

Jagers, P. (1975), Branching Processes with Biological Applications, John Wiley, Chichester.

Janson S. (1993), Multicyclic components in a random graph process, Random Structures and Algorithms 4 (1993), 71-84.

Janson S., Knuth D. E., Łuczak T., and Pittel B. (1993), The birth of the giant component, Random Structures and Algorithms 4 (1993), 233-358.

Kesten, H. and Schonmann, R. H. (1990), Behavior in large dimensions of the Potts and Heisenberg models, Reviews in Mathematical Physics 1, 147-182.

Łuczak T., Pittel B., and Wierman J. C. (1994), The structure of a random graph at the point of the phase transition, Transactions of the American Mathematical Society 341, 721-748.

Pittel, B. (1990), On tree census and the giant component of sparse random graphs, Random Structures and Algorithms 1, 311-341.

Thompson, C. J. (1972), Mathematical Statistical Mechanics, Macmillan, New York.

Whittle, P. (1986), Systems in Stochastic Equilibrium, John Wiley, Chichester.

Whittle, P. (1994), Polymer models and generalized Potts-Kasteleyn models, Journal of Statistical Physics 75, 1063-1092.

Wu, F. Y. (1982), The Potts model, Reviews in Modern Physics 54, 235-268.

Béla Bollobás, DPMMS, University of Cambridge, 16 Mill Lane, Cambridge CB2 1SB, UK

E-mail address: b.bollobas@pmms.cam.ac.uk

Geoffrey Grimmett, Statistical Laboratory, University of Cambridge, 16 Mill

Lane, Cambridge CB2 $1 \mathrm{SB}, \mathrm{UK}$

E-mail address: g.r.grimmett@statslab.cam.ac.uk

Svante Janson, Department of Mathematics, Uppsala University, PO Box 480, S-751 06 Uppsala, SwEDEN

E-mail address: svante.janson@math.uu.se 